\title{
Collective generation of quantum states of light by entangled atoms
}

\author{
D. Porras* and J. I. Cirac ${ }^{\dagger}$ \\ Max-Planck-Institut für Quantenoptik, Hans-Kopfermann-Strasse 1, Garching, D-85748, Germany
}

(Received 20 August 2008; published 12 November 2008)

\begin{abstract}
We present a theoretical framework to describe the collective emission of light by entangled atomic states. Our theory applies to the low-excitation regime, where most of the atoms are initially in the ground state, and relies on a bosonic description of the atomic excitations. In this way, the problem of light emission by an ensemble of atoms can be solved exactly, including dipole-dipole interactions and multiple light scattering. Explicit expressions for the emitted photonic states are obtained in several situations, such as those of atoms in regular lattices and atomic vapors. We determine the directionality of the photonic beam, the purity of the photonic state, and the renormalization of the emission rates. We also show how to observe collective phenomena with ultracold atoms in optical lattices and how to use these ideas to generate photonic states that are useful in the context of quantum information.
\end{abstract}

DOI: 10.1103/PhysRevA.78.053816

PACS number(s): 42.50.Nn, 42.50.Ex, 37.10.Jk, 03.67.Hk

\section{INTRODUCTION}

In the last years, the field of atomic, molecular, and optical physics has witnessed an impressive advance in the development of setups to trap atoms under different conditions, such as, for example, ions in electromagnetic traps and ultracold atoms in optical lattices. Furthermore, the quantum state of these systems may be engineered by performing quantum operations such as as quantum gates between ions [1] or the excitation of neutral atoms under the dipole blockade $[2,3]$. In this way one can create deterministically collective entangled states, such as the completely symmetric states with a single excited atom ( $|\mathrm{W}\rangle$ states) [4]. In addition, those are systems where atoms can be coupled to light in a very controlled way. Since some of the atomic entangled states which may be created in those setups play an important role in the description of the interaction of atomic ensembles with light, an important question arises: namely, can we use our control on the states of trapped atoms to generate useful quantum states of light? If so, which are the properties of such states in terms of photon directionality, purity, or photon entanglement?

A preliminary study of these ideas was recently presented in [5]. The understanding of this problem requires a theoretical framework to describe the emission of light by collective atomic states under a variety of trapping conditions. Related problems have been indeed a subject of investigation since the seminal work by Dicke [6]. Nevertheless, the experimental systems that we have in mind share a few peculiarities which demand a new approach for their description. First, ultracold atoms are trapped in ordered arrangements such as Coulomb crystals or optical lattices, where the distance between atoms, $d_{0}$, is comparable to the wavelength of the light, $\lambda$. This situation is very different from Dicke superradiance, where $\lambda$ is larger than the size of the whole system. It also differs from the case of crystals, where $\lambda \gg d_{0}$ as considered, for example, in [7]. Also, we study a situation in

\footnotetext{
*Diego.Porras@mpq.mpg.de

†Inacio.Cirac@mpq.mpg.de
}

which atoms are initialized in a collective state, which is not necesarilly created by the absorption of a photon. Thus, rather than the more traditional description in terms of light scattering, we need a theoretical model of the mapping between atomic collective excitations and photons.

In its more general form the above-described situation poses a very complicated many-body problem. A crucial simplification is achieved by considering the low-excitation sector of the atomic Hilbert space, which in the HolsteinPrimakoff (HP) approximation can be described in terms of bosonic spin waves [8]. This approach has been used in previous works, for example, to study Dicke superradiance [9], slow propagation of light [10], and atom-light interfaces with atomic vapors [11]. Also, the emission of light by ensembles of harmonic oscillators was studied in [12], although in a different regime of trapping conditions than those considered here.

In this paper we make use of the HP approximation to describe the collective emission of light as a mapping between spin-wave excitations and photons. For atoms placed at fixed positions this mapping is a Gaussian completely positive map [13], and we present a method to get explicit expressions of the photonic modes into which light is emitted. Furthermore, we extend this formalism to study the case of atomic vapors. We obtain the following results: (i) For atoms trapped in a regular lattice, there is a regime in which photons may be emitted in a collimated beam, which requires that $\lambda>2 d_{0}$. In this regime, there is a renormalization of the emission rates, leading to a classification of the lowexcitation atomic Hilbert space in terms of superradiant and subradiant spin waves. Superradiant states decay with a rate that is enhanced by a factor $\chi$, which depends on the dimensionality of the lattice. In one dimension and 3D we determine the values $\chi_{1 D} \propto \lambda / d_{0}$ and $\chi_{3 D} \propto\left(\lambda / d_{0}\right)^{2}\left(L / d_{0}\right)$, respectively, with $L$ the length of the lattice. This effect is related but not equivalent to Dicke superradiance. (ii) In the case of atomic vapors the collective character in the emission of light is determined by $\chi_{\mathrm{en}} \propto N(\lambda / L)^{2}$, where $N$ is the total number of atoms. The directional regime requires $\chi_{\mathrm{en}} \gg 1$, and the emission rate is enhanced by $\chi_{\mathrm{en}}$. (iii) Some of these effects, such as the renormalization of the emission rates and the directionality, could be observed in a relatively simple 


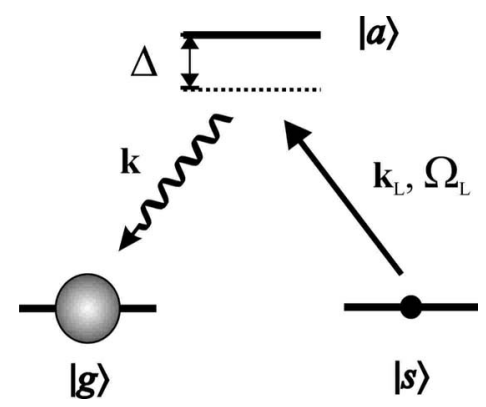

FIG. 1. $\Lambda$ scheme.

experiment with atoms in optical lattices. (iv) By making use of coherent effects, photonic entangled states could be generated by trapped ions or neutral atoms. Photons that are generated in this way could be collimated and in a pure state and thus be useful in the context of quantum information.

\section{THEORETICAL FRAMEWORK}

Our first task is to describe the collective emission of light by an ensemble of atoms. First, we focus on the situation in which atoms are placed at fixed positions. At the end of the section we discuss the effect of atomic motion.

\section{A. Atom-photon map in the Holstein-Primakoff approximation}

We consider an ensemble of $N$ atoms trapped by harmonic potentials with trapping frequency $\nu$ and internal levels forming a $\Lambda$ scheme (see Fig. 1). Two ground states $|g\rangle$ and $|s\rangle$ are coupled by means of a laser with Rabi frequency $\Omega_{\mathrm{L}}$ and wave vector $\mathbf{k}_{\mathrm{L}}$ through an auxiliary level $|a\rangle$. We define atomic operators $\sigma_{j}=|g\rangle_{j}\langle s|$ and $\sigma_{j}^{\mathrm{ss}}=|s\rangle_{j}\langle s|$, which fulfill the commutation relations

$$
\left\langle\left[\sigma_{j}, \sigma_{l}^{\dagger}\right]\right\rangle=\delta_{j, l}\left(1-2\left\langle\sigma_{j}^{\mathrm{ss}}\right\rangle\right)
$$

Under the condition that the excitation probability of each atom be low, $\left\langle\sigma_{j}^{\text {ss }}\right\rangle \ll 1$, we replace atomic operators by HP bosons, $\sigma_{j} \rightarrow b_{j}$.

The HP approximation allows us to recast the atom-light Hamiltonian as a bosonic quadratic Hamiltonian. After adiabatic elimination of the upper level $|a\rangle$, our system is described by

$$
H=H_{0}+H_{\mathrm{lm}}+H_{\mathrm{mot}} .
$$

$H_{0}=\Sigma_{\mathbf{k}} \omega_{k} a_{\mathbf{k}}^{\dagger} a_{\mathbf{k}}$, with $\omega_{k}=c k$. We consider, for the sake of clarity, a scalar model for the electromagnetic field, since our conclusions do not change when including the dipole pattern, as we show later. However, the photon polarization can be included straightforwardly in our formalism. $H_{1 \mathrm{~m}}$ is the atomlight interaction Hamiltonian in the rotating-wave approximation [14] (we set $\hbar=1$ ),

$$
H_{\mathrm{lm}}=\sum_{j, \mathbf{k}} g_{k} b_{j}^{\dagger} a_{\mathbf{k}} e^{i\left(\mathbf{k}-\mathbf{k}_{\mathrm{L}}\right) \cdot \mathbf{r}_{j}+i \omega_{\mathrm{L}} t}+\text { H.c. }
$$

$$
g_{k}=\frac{\Omega_{\mathrm{L}}}{2 \Delta} \frac{\hbar \omega_{k}}{2 \epsilon_{0} V} d_{\mathrm{ga}} .
$$

$V$ is the quantization volume of the electromagnetic field, $\epsilon_{0}$ the vacuum permitivity, and $d_{g a}$ the dipole matrix element of the $|g\rangle-|a\rangle$ transition. $H_{\text {mot }}$ is the Hamiltonian describing the motion of the atoms during the emission process, whose energy scale is given typically by the trapping frequency $\nu$. Equation (3) is obtained under the assumption $\Delta \gg \Omega_{\mathrm{L}}, \nu \bar{n}$, with $\bar{n}$ the mean vibrational occupation number. In the resonant case $\Delta=0$, the validity of the adiabatic elimination of $|a\rangle$ requires that the decay rate from $|a\rangle$ to $|g\rangle, \Gamma_{\mathrm{ag}}$, satisfy $\Gamma_{\mathrm{ag}} \gg \Omega_{\mathrm{L}}, \nu \bar{n}$, and a similar coupling as (3) is obtained. The spontaneous decay of $|a\rangle$ back to $|s\rangle$ is neglected in the forecoming analysis. This is justified either when collective effects enhance the $|a\rangle-|g\rangle$ channel or by choosing $|a\rangle-|g\rangle$ to be a cycling transition (see Appendix D). We deal first with the simplest case, in which atoms are placed at fixed positions and the motion of the atoms can be neglected. This is a good approximation in the Lamb-Dicke regime $\left(k_{\mathrm{L}} x_{0}\right)^{2}(2 \bar{n}+1)$ $\ll 1$, where $x_{0}$ is the ground-state size in the harmonic trap. The effect of the motion is considered in the last subsection.

This work relies on the observation that $H_{0}+H_{1 \mathrm{~m}}$ generates a beam-splitter transformation between atomic states and photons. The evolution of the atom-photon system can thus be understood in terms of a Gaussian completely positive map [13]. In the low-excitation regime, any atomic state can be expressed in terms of bosonic excitations,

$$
|\Psi\rangle_{\text {at }}=\sum_{n_{1}, \ldots, n_{N}} \Psi_{n_{1}, \ldots, n_{N}} \frac{\left(b_{1}^{\dagger}\right)^{n_{1}}}{\sqrt{n_{1} !}} \ldots \frac{\left(b_{N}^{\dagger}\right)^{n_{N}}}{\sqrt{n_{N} !}}|0\rangle_{\text {at }} .
$$

We consider the initial state

$$
|\psi(0)\rangle=|\Psi\rangle_{\mathrm{at}}|0\rangle_{\mathrm{ph}},
$$

where $|0\rangle_{\mathrm{ph}}$ is the photon vacuum. Our goal is to find the photon state $|\Phi\rangle_{\mathrm{ph}}$ at a time $t$ longer than the atomic decay time,

$$
|\psi(t)\rangle=\mathcal{U}(t)|\psi(0)\rangle=|0\rangle_{\mathrm{at}}|\Phi\rangle_{\mathrm{ph}},
$$

where $\mathcal{U}(t)=e^{-i H t}$ and $H$ is the total atom-system Hamiltonian. Together with the beam splitter form of (3) this implies that the problem is reduced to finding the exact form of the transformation,

$$
\mathcal{U}(t) b_{j}^{\dagger} \mathcal{U}(t)^{\dagger}=\sum_{\mathbf{k}} g_{j \mathbf{k}}(t) a_{\mathbf{k}}^{\dagger}+\sum_{l} h_{j l}(t) b_{l}^{\dagger} .
$$

Taking into account that $\mathcal{U}(t)^{\dagger}|\mathrm{vac}\rangle=0$, with $|\mathrm{vac}\rangle=|0\rangle_{\mathrm{at}}|0\rangle_{\mathrm{ph}}$, then $g_{j \mathbf{k}}(t)$ can be found by using the relation

$$
g_{j \mathbf{k}}(t)=\left\langle\operatorname{vac}\left|a_{\mathbf{k}} \mathcal{U}(t) b_{j}^{\dagger}\right| \operatorname{vac}\right\rangle=\left\langle\operatorname{vac}\left|a_{\mathbf{k}}(t) b_{j}^{\dagger}\right| \operatorname{vac}\right\rangle,
$$

where $a_{\mathbf{k}}(t)$ is the photon operator in the Heisenberg picture. Also,

$$
h_{j l}(t)=\left\langle\operatorname{vac}\left|b_{l} \mathcal{U}(t) b_{j}^{\dagger}\right| \operatorname{vac}\right\rangle=\left\langle\operatorname{vac}\left|b_{l}(t) b_{j}^{\dagger}\right| \operatorname{vac}\right\rangle,
$$

which shows that $h_{j l}(t)=0$ for $t$ longer than the atomic decay time. $g_{j \mathbf{k}}(t)$ is thus the only interesting term, since it determines the photon mode into which light is radiated. Its determination can be readily done by means of the Heisenberg 
equation of motion for the photonic operators, which yields

$$
\begin{aligned}
g_{j \mathbf{k}}(t)= & -i g_{k} e^{-i \omega_{k} t} \sum_{l} \int_{0}^{t} d \tau \\
& \times e^{-i\left(\mathbf{k}-\mathbf{k}_{\mathrm{L}}\right) \cdot \mathbf{r}_{l}+i\left(\omega_{k}-\omega_{\mathrm{L}}\right) \tau}\left\langle\operatorname{vac}\left|b_{l}(\tau) b_{j}^{\dagger}\right| \mathrm{vac}\right\rangle .
\end{aligned}
$$

This equation is our starting point for the exact determination of the photonic modes.

\section{B. Photonic modes}

To find the mapping between atomic and photonic modes, we need to solve the master equation to calculate the time evolution of the atomic correlator in Eq. (10). In general, this is a difficult many-body problem, but in the bosonic limit it can be described exactly. In terms of HP bosons, the master equation which describes the atomic dynamics reads [15]

$$
\frac{d \rho}{d t}=\sum_{i, j}\left(J_{i j} b_{j} \rho b_{i}^{\dagger}-J_{i j} b_{i}^{\dagger} b_{j} \rho+\text { H.c. }\right),
$$

where $J_{i j}$ include multiple light scattering and dipole-dipole interactions,

$$
J_{i j}=\sum_{\mathbf{k}} g_{k}^{2} \int_{0}^{\infty} e^{i\left(\omega_{k}-\omega_{\mathrm{L}}\right) \tau+i\left(\mathbf{k}-\mathbf{k}_{\mathrm{L}}\right) \cdot\left(\mathbf{r}_{i}-\mathbf{r}_{j}\right)} d \tau .
$$

This expression is evaluated by using the identity $\int d \tau e^{i \omega \tau}$ $=\pi \delta(\omega)+i \mathrm{P}(1 / \omega)$, which yields the result

$$
\begin{gathered}
J_{i i}=\frac{1}{2} \bar{\Gamma}, \\
J_{i j}=\frac{1}{2} \bar{\Gamma} e^{-i \mathbf{k}_{\mathrm{L}}\left(\mathbf{r}_{i}-\mathbf{r}_{j}\right)}\left(\frac{\sin \left(k_{\mathrm{L}}\left|\mathbf{r}_{i}-\mathbf{r}_{j}\right|\right)}{k_{\mathrm{L}}\left|\mathbf{r}_{i}-\mathbf{r}_{j}\right|}-i \frac{\cos \left(k_{\mathrm{L}}\left|\mathbf{r}_{i}-\mathbf{r}_{j}\right|\right)}{k_{\mathrm{L}}\left|\mathbf{r}_{i}-\mathbf{r}_{j}\right|}\right) \\
\times(i \neq j),
\end{gathered}
$$

where

$$
\bar{\Gamma}=\frac{1}{3 \pi}\left(\frac{\Omega_{\mathrm{L}}}{2 \Delta}\right)^{2} \frac{\omega_{\mathrm{L}}^{3}}{\epsilon_{0} c^{3}} d_{\mathrm{ga}}^{2}
$$

is the single-atom decay rate. The inclusion of the photon polarization would change the spatial dependence of the couplings $J_{i j}$. However, as we show later, the collective phenomena would be the same. Note that we are not including the single-atom Lamb shift, which may be simply absorbed into the laser frequency $\omega_{\mathrm{L}}$. Since Eq. (11) is quadratic in bosonic operators, it is readily solved by defining eigenmodes which diagonalize the atomic quantum dynamics in the lowexcitation limit,

$$
\begin{gathered}
b_{j}=\sum_{n} \mathcal{M}_{j n} b_{n}, \\
\left(\mathcal{M}^{-1} J \mathcal{M}\right)_{n m}=J_{n} \delta_{n, m} .
\end{gathered}
$$

The matrix $J_{i j}$ is not Hermitian, and thus canonical commutation relations are not conserved, $\left[b_{n}, b_{m}^{\dagger}\right] \neq \delta_{n, m}$. However, the evolution of averages takes a simple form given by

$$
\left\langle b_{l}(\tau)\right\rangle=\sum_{n} \mathcal{M}_{l n} e^{-J_{n} \tau}\left\langle b_{n}(0)\right\rangle
$$

Thus, the spin-wave dynamics is governed by the eigenvalues $J_{n}$. The latter contain the collective decay rates $\Gamma_{n}$ and the collective energy shifts $\Delta_{n}$,

$$
\Gamma_{n}=2 \operatorname{Re}\left(J_{n}\right), \quad \Delta_{n}=2 \operatorname{Im}\left(J_{n}\right) .
$$

Conservation of the trace under the transformation (15) leads to the sum rules

$$
\sum_{n} \Gamma_{n}=N \bar{\Gamma}, \quad \sum_{n} \Delta_{n}=0
$$

Note that whenever collective effects induce a renormalization of $\Gamma_{n}$, the sum rule implies the existence of super- and subradiant states.

By application of (15) and (16) and the quantum regression theorem, we determine the two-time atomic average in (10). In the limit that $t \gg 1 / \Gamma_{n}$ we get

$$
g_{j \mathbf{k}}(t)=i e^{-i \omega_{k} t} g_{\mathbf{k}} \sum_{l n} \frac{\left(\mathcal{M}^{-1}\right)_{n j} e^{-i\left(\mathbf{k}-\mathbf{k}_{\mathrm{L}}\right) \cdot \mathbf{r}_{l}} \mathcal{M}_{l n}}{i\left(\omega_{k}-\omega_{\mathrm{L}}\right)-J_{n}},
$$

which yields the explicit form of the atom-photon mapping. By means of this relation it is possible to determine the many-photon state emitted by any initial atomic state like (4).

To get insight into the characteristics of the photonic states emitted by the collective atomic states, from now on we focus on the mapping to a single photon. To clarify the notation, let us define $\left|\Psi_{n}\right\rangle_{\text {at }}$, the $n$-spin-wave state with a single excitation,

$$
\begin{aligned}
\left|\Psi_{n}\right\rangle_{\mathrm{at}} & =\frac{1}{\mathcal{N}_{n}} \sum_{j} \mathcal{M}_{j n} b_{j}^{\dagger}|0\rangle_{\mathrm{at}}, \\
\mathcal{N}_{n} & =\sqrt{\sum_{j} \mathcal{M}_{j n}^{*} \mathcal{M}_{j n} .}
\end{aligned}
$$

$\mathcal{N}$ has to be included due to the non-Hermiticity of $J_{i j}$. We determine $\left|\Phi_{n}\right\rangle_{\mathrm{ph}}$, the single-photon state into which $\left|\Psi_{n}\right\rangle_{\text {at }}$ is mapped,

$$
\begin{gathered}
\left|\Phi_{n}(t)\right\rangle_{\mathrm{ph}}=\sum_{\mathbf{k}} \phi_{n, \mathbf{k}}(t) a_{\mathbf{k}}^{\dagger}|0\rangle_{\mathrm{ph}}, \\
\phi_{n, \mathbf{k}}(t)=i \frac{g_{k} e^{-i \omega_{k} t}}{\mathcal{N}_{n}} \sum_{l} \frac{e^{-i\left(\mathbf{k}-\mathbf{k}_{\mathrm{L}}\right) \cdot \mathbf{r}_{l} \mathcal{M}_{l n}}}{i\left(\omega_{k}-\omega_{\mathrm{L}}\right)-J_{n}} .
\end{gathered}
$$

Although these results allow one to solve exactly the problem of collective emission of light including the effects of reabsorption, aditional insight can be gained by considering the case of a system with periodic boundary conditions. This will be a good approximation for a finite system, provided that the number of atoms in the volume is much larger than in the surface-that is, $L / d_{0} \gg 1$. In this case we get the matrices 


$$
\mathcal{M}_{j n}=\frac{1}{\sqrt{N}} e^{i \mathbf{K}_{n} \cdot \mathbf{r}_{j}}
$$

The spin-wave state $n$ is then defined like

$$
\left|\Psi_{n}\right\rangle_{\mathrm{at}}=\frac{1}{\sqrt{N}} \sum_{j} e^{i \mathbf{K}_{n} \cdot \mathbf{r}_{j}} b_{j}^{\dagger}|0\rangle_{\mathrm{at}} .
$$

The vector $\mathbf{K}_{n}$ is the momentum of the collective atomic state. The resulting photonic mode is defined by the expression

$$
\phi_{n, \mathbf{k}}(t)=i g_{k} e^{-i \omega_{k} t} \frac{\frac{1}{\sqrt{N}} \sum_{l} e^{-i\left(\mathbf{k}-\mathbf{k}_{\mathrm{L}}-\mathbf{K}_{n}\right) \cdot \mathbf{r}_{l}}}{i\left(\omega_{k}-\omega_{\mathrm{L}}\right)-J_{n}},
$$

such that collective effects and dipole-dipole interactions enter through the dependence of the collective emission rate $J_{n}$ on the mode number $n$. Note that by using periodic boundary conditions, the matrix $\mathcal{M}$ defines an unitary transformation, and thus the sets $\left|\Psi_{n}\right\rangle_{n=1, \ldots, N}$ and $\left|\Phi_{n}\right\rangle_{n=1, \ldots, N}$ form an orthogonal basis of spin waves and photonic modes, respectively.

\section{Angular photon number distribution}

We determine now the properties of the emitted photonic modes. In particular, let us define

$$
I(\Omega)=\frac{V}{(2 \pi)^{3}} \int_{0}^{\infty}\left\langle a_{\mathbf{k}}^{\dagger} a_{\mathbf{k}}\right\rangle k^{2} d k,
$$

the average photon number per solid angle. Consider an initial atomic state with a single excitation,

$$
|\psi\rangle_{\mathrm{at}}=\sum_{j} \psi_{j} b_{j}^{\dagger}|0\rangle_{\mathrm{at}} .
$$

The emitted photon distribution is

$$
\begin{gathered}
I(\Omega)=\sum_{j, j^{\prime}} \psi_{j}^{*} I_{j j^{\prime}}(\Omega) \psi_{j^{\prime}}, \\
I_{j j^{\prime}}(\Omega)=\frac{V}{(2 \pi)^{3}} \int_{0}^{\infty} g_{j, \mathbf{k}}^{*} g_{j^{\prime}, \mathbf{k}} k^{2} d k .
\end{gathered}
$$

Upon substitution of (19) in the expression for $I_{j j^{\prime}}(\Omega)$ and under the condition that $\Gamma_{n} / c \ll 1 / L$ (see Appendix A), we get

$$
\begin{gathered}
I_{j j^{\prime}}(\Omega)=\frac{\bar{\Gamma}}{4 \pi} \sum_{n, n^{\prime}} B(\Omega)_{j n} \frac{1}{\bar{J}_{n}^{*}+\bar{J}_{n^{\prime}}} B(\Omega)_{j^{\prime} n^{\prime}}, \\
B(\Omega)_{j n}=\left(\mathcal{M}^{-1}\right)_{n j}^{*} \sum_{l} \mathcal{M}_{l n}^{*} e^{i\left(k_{\mathrm{L}} \mathbf{n}_{\Omega^{-}} \mathbf{k}_{\mathrm{L}}\right) \cdot \mathbf{r}_{l}},
\end{gathered}
$$

where $\mathbf{u}_{\Omega}$ is a unit vector pointing in the direction of the solid angle $\Omega$. In spherical coordinates, $\Omega$ is determined by $(\theta, \phi)$, such that $\mathbf{u}_{\Omega}=(\sin \theta \cos \phi, \sin \theta \sin \phi, \cos \theta)$.

The general recipe for calculating the photon distribution probability involves the following steps: (i) Calculate the coefficients of the master equation and find the eigenvalues and eigenvectors of $J_{i j}$. (ii) Use Eq. (28) to calculate $I_{i j}$. (iii) Use the latter to calculate the emission spectrum with the wave function of any given initial atomic state expressed in terms of bosonic spin waves. In the plane-wave approximation (22) we can get closed expressions for the photon distribution. We focus again on the single-photon case and define $I_{n}(\Omega)$ as the photon number distribution corresponding to the photonic mode emitted by $\left|\Psi_{n}\right\rangle_{\mathrm{at}}$. By using Eq. (28) we get

$$
I_{n}(\Omega)=\frac{1}{4 \pi} \frac{\bar{\Gamma}}{\Gamma_{n}} \sum_{j, j^{\prime}} e^{i\left(\mathbf{k}_{\Omega^{\prime}}-\mathbf{k}_{\mathrm{L}}-\mathbf{K}_{n}\right) \cdot\left(\mathbf{r}_{j}-\mathbf{r}_{j^{\prime}}\right)} .
$$

This result has a clear interpretation in terms of interference of light emitted by the atomic system [16,17]. Equation (29) not only allows one to calculate the angular emission probabilty, but also, due to the normalization condition $\int I_{n}(\Omega) d \Omega=1$,

$$
\frac{\Gamma_{n}}{\bar{\Gamma}}=\int d \Omega \frac{1}{4 \pi} \sum_{j, j^{\prime}} e^{i\left(\mathbf{k}_{\Omega^{\prime}}-\mathbf{k}_{\mathrm{L}}-\mathbf{K}_{n}\right) \cdot\left(\mathbf{r}_{j}-\mathbf{r}_{j^{\prime}}\right)} .
$$

This expression provides us with a simple way to determine the collective rates under the plane-wave approximation.

\section{Effects of the atomic motion}

Finally, we discuss the effect of the atomic motion on the light emission. In the most general case, the inclusion of the motional degrees of freedom poses a very complicated problem which goes beyond the scope of this work. Two time scales determine this problem. The first is $\tau_{\text {mot }}$, the time scale of the motion of atoms in the trap. In the case of trapped particles, $\tau_{\text {mot }}=1 / \nu$, with $\nu$ the trapping frequency. We can extend this discussion to the case of atomic vapors and consider that in this case, $\tau_{\text {mot }}=L / v$, with $L$ the length of the sample and $v$ the atom velocity. $\tau_{\text {mot }}$ is to be compared with the radiative decay time $1 / \bar{\Gamma}$, or more specifically, the set of collective decay times $1 / \Gamma_{n}$. Based on the comparison between these time scales we define two limits in which the application of our theoretical framework is particularly straightforward.

(i) Slow-motion limit $\tau_{\text {mot }} \gg 1 / \Gamma_{n}$. Since the emission process is much faster than the motion of the particles, we can assume that atomic positions are frozen. The system is in the initial state

$$
|\psi(0)\rangle=|\Psi\rangle_{\mathrm{at}}|0\rangle_{\mathrm{ph}}\left|\Psi_{m}\right\rangle_{\mathrm{mot}},
$$

where $\Psi_{\mathrm{m}}\left(\mathbf{r}_{1}, \ldots, \mathbf{r}_{N}\right)$ is the initial wave function in terms of the atomic positions, which in this limit does not evolve during the emission time. The atom-photon mapping can be still applied to this system by solving it for each value of the atomic positions,

$$
|\Psi\rangle_{\text {at }}|0\rangle_{\text {at }} \rightarrow|0\rangle_{\text {at }}\left|\Phi^{\mathbf{r}_{1}, \ldots, \mathbf{r}_{N}}\right\rangle_{\mathrm{ph}},
$$

where $\left|\Phi^{\mathbf{r}_{1}, \ldots, \mathbf{r}_{N}}\right\rangle_{\mathrm{ph}}$ is the photonic state obtained under the assumptions that atoms are located at positions $\mathbf{r}_{1}, \ldots \mathbf{r}_{N}$. Any photonic observable $O$ is then obtained upon averaging with 
respect to the wave function $\Psi_{\mathrm{m}}$-for example,

$$
\langle O\rangle=\sum_{\mathbf{r}_{1}, \ldots, \mathbf{r}_{N}}\left\langle\Phi^{\mathbf{r}_{1}, \ldots, \mathbf{r}_{N}}|O| \Phi^{\left.\mathbf{r}_{1}, \ldots, \mathbf{r}_{N}\right\rangle}\left|\Psi_{m}\left(\mathbf{r}_{1}, \ldots, \mathbf{r}_{N}\right)\right|^{2} .\right.
$$

This method can be readly extended to the case of a mixed motional state.

(ii) Fast-motion limit $\tau_{\text {mot }} \ll 1 / \Gamma_{n}$. In this case, we are assuming that trapped atoms move along the sample in a time that is smaller than the emission time. This case is particularly relevant, since it describes hot atomic vapors. We notice first that condition $\tau_{\text {mot }} \ll 1 / \Gamma_{n}$ implies that the atomic positions are not correlated with the atomic operators in Eq. (10). Thus, we can describe the atomic radiative decay indepently of the evolution of atomic positions $\mathbf{r}_{l}$ in (10). This can be done by using a master equation with averaged coefficients,

$$
J_{i j}=\pi \sum_{\mathbf{k}} g_{k}^{2} \delta\left(\omega_{k}-\omega_{\mathrm{L}}\right)\left\langle e^{i\left(\mathbf{k}-\mathbf{k}_{\mathrm{L}}\right) \cdot\left(\mathbf{r}_{i}-\mathbf{r}_{j}\right)}\right\rangle_{\mathrm{mot}} \cdot
$$

In the case of a harmonic trap, $\mathbf{r}_{i}$ are position operators and $\langle\cdots\rangle_{\text {mot }}$ is the average with the atomic motional state. This situation can be extended to describe hot atomic vapors by replacing the atomic positions $\mathbf{r}_{i}$ by a set of random variables with a given probability distribution, $\rho_{\mathrm{m}}\left(\mathbf{r}_{1}, \ldots, \mathbf{r}_{N}\right)$, and performing the corresponding average to get $J_{i j}$.

Once the atomic dynamics is solved, our results on the atom-photon mapping can be used to describe the emitted photons. A general atomic state is mapped now onto a mixed photonic state. For example, consider the atomic state $\left|\Psi_{n}\right\rangle_{\text {at }}$, defined by Eq. (20), which is obtained by the diagonalization of the master equation with (61). The atom-photon mapping yields the following photon density matrix after the emission process:

$$
\rho^{\mathrm{ph}}(t)=\sum_{\mathbf{k}, \mathbf{k}^{\prime}}\left\langle\phi_{n, \mathbf{k}}(t) \phi_{n, \mathbf{k}^{\prime}}^{*}(t)\right\rangle_{\mathrm{mot}} a_{\mathbf{k}}^{\dagger}|0\rangle_{\mathrm{ph}}\langle 0| a_{\mathbf{k}},
$$

and this expression is easily generalized to the multiphoton case. Note that the solution of the fast-motion limit seems similar to the case of slow motion. However, the crucial difference is that in the fast case, we are allowed to solve the radiative emission problem and to perform subsequently the spatial average in (35). We will study in more detail this situation later in the case of the collective emission properties of atomic ensembles.

\section{ATOM-PHOTON MAPPING IN A SQUARE LATTICE}

The situation in which atoms are arranged in a crystal is found in experimental setups such as ultracold atoms in optical lattices and Coulomb crystals of trapped ions. The results presented in the previous section are applied here to study the collective emission process in these systems. We obtain analytical results by using the plane-wave approximation.

\section{A. General discussion}

Let us study for concreteness the case of $1 \mathrm{D}$ or $3 \mathrm{D}$ square lattices, although our results are easily generalized to differ- ent lattice geometries. Assuming periodic boundary conditions, the allowed wave vectors are

$$
\mathbf{K}_{\mathbf{n}}=\frac{2 \pi}{d_{0}} \sum_{\alpha} \frac{n_{\alpha}}{N_{\alpha}} \hat{\alpha}
$$

In the $1 \mathrm{D}$ case we consider that the atom chain is aligned in the $z$ direction. Thus, in (36) and the forecoming expressions, $\alpha$ runs over $\alpha=z$ in $1 \mathrm{D}$ and $\alpha=x, y, z$ in 3D. $\hat{\alpha}$ is a unit vector in the direction $\alpha$, and $N_{\alpha}$ is the number of atoms along $\hat{\alpha}$. Considering, for concreteness, the case of even $N_{\alpha}$, each wave vector is determined by the set of integers $n_{a}$ $=-N_{\alpha} / 2, \ldots, N_{\alpha} / 2-1$. By applying Eq. (29) we determine the photon number angular distribution for the photonic state emitted by a spin-wave excitation with momentum $\mathbf{K}_{\mathbf{n}}$,

$$
I_{\mathbf{n}}(\Omega)=\frac{1}{4 \pi} \frac{\bar{\Gamma}}{\Gamma_{\mathbf{n}}} \frac{1}{N} \prod_{\alpha} \frac{\sin ^{2}\left[\left(k_{\mathrm{L}} \mathbf{u}_{\Omega}^{\alpha}-\mathbf{k}_{\mathrm{L}}^{\alpha}-\mathbf{K}_{\mathbf{n}}^{\alpha}\right) d_{0} N_{\alpha} / 2\right]}{\sin ^{2}\left[\left(k_{\mathrm{L}} \mathbf{u}_{\Omega}^{\alpha}-\mathbf{k}_{\mathrm{L}}^{\alpha}-\mathbf{K}_{\mathbf{n}}^{\alpha}\right) d_{0} / 2\right]} .
$$

$I_{\mathbf{n}}(\Omega)$ shows a series of diffraction maxima at solid angles $\Omega$ at which the $\sin ^{2}$ function in the denominator vanishes. Note that by including the photon polarization, we would have obtained an additional function of $\Omega$ multiplying the photon distribution $I_{n}(\Omega)$, which would correspond to the singleatom dipole pattern. The latter would induce the suppression of diffraction peaks if they are in a direction forbidden by the dipole pattern. Since we are specifically interested in collective effects, we do not consider this effect in the discussion that follows.

To get a quantitative description of the photon distribution we notice first that

$$
\frac{1}{N} \frac{\sin ^{2}(N x)}{\sin ^{2}(x)} \approx \sum_{m} f_{N}(x-m \pi), \quad \text { if } N \gg 1,
$$

where the function $f_{N}(x)$ describes the shape of each of the diffraction peaks,

$$
\begin{gathered}
f_{N}(x)=N \operatorname{sinc}^{2}(N x), \quad-\pi / 2<x<\pi / 2, \\
f_{N}(x)=0, \quad \text { otherwise. }
\end{gathered}
$$

Thus, in the limit $N_{\alpha} \gg 1$, one can approximate the emission probability as a sum over Bragg scattering contributions,

$$
I_{\mathbf{n}}(\Omega)=\sum_{\mathbf{m}} I_{\mathbf{n}}^{[\mathbf{m}]}(\Omega),
$$

$$
I_{\mathbf{n}}^{[\mathbf{m}]}(\Omega)=\frac{1}{4 \pi} \frac{\bar{\Gamma}}{\Gamma_{\mathbf{n}}} \prod_{\alpha} f_{N_{\alpha}}\left(\frac{k_{\mathrm{L}} d_{0} \mathbf{n}_{\Omega}^{\alpha}}{2}-\frac{\mathbf{k}_{\mathrm{L}}^{\alpha} d_{0}}{2}-\frac{n_{\alpha}}{N_{\alpha}} \pi+m_{\alpha} \pi\right) .
$$

Each term in the sum is labeled by the vector $\mathbf{m}$ and corresponds to a different diffraction peak. The probability that the spin wave $\mathbf{n}$ emits a photon in the $\mathbf{m}$ diffraction peak is given by

$$
p_{\mathbf{n}}^{[\mathbf{m}]}=\int d \Omega I_{\mathbf{n}}^{[\mathbf{m}]}(\Omega) .
$$


Equation (40) has a clear interpretation in terms of momentum conservation. $I_{\mathbf{n}}(\Omega)$ has a maximum whenever there is a value of $\mathbf{m}$ such that

$$
k_{\mathrm{L}} \mathbf{u}_{\Omega}^{\alpha}=\mathbf{k}_{\mathrm{L}}^{\alpha}+n_{\alpha} \frac{2 \pi}{d_{0} N_{\alpha}}+m_{\alpha} \frac{2 \pi}{d_{0}}, \quad \forall \alpha .
$$

That is, the linear momentum of the emitted photon has to match the sum of three contributions: the momentum of the incident laser, $\mathbf{k}_{\mathrm{L}}$; the initial momentum of the spin wave, $\mathbf{n} 2 \pi /\left(d_{0} N_{\alpha}\right)$; and the contribution from the lattice periodicity, which enters through the reciprocal wave vector $\mathbf{m} 2 \pi / d_{0}$. In $1 \mathrm{D}$, condition (42) has to be satisfied only by the $\alpha=z$ component of these vectors; that is, only the projection of the momentum on the chain is conserved. In $3 \mathrm{D}$, on the contrary, the equality has to be satisfied by all vector components. Relation (42) determines the maxima in the emission pattern depending on $d_{0}$ and $\lambda$, but it also determines the collective rates, through the normalization condition on $I_{\mathbf{n}}(\Omega)$; see Eq. (30).

Since $-1 \leqslant \mathbf{u}_{\Omega}^{\alpha} \leqslant 1$, if $\lambda>2 d_{0}$, there is at most a single value of $\mathbf{m}$, for which condition (42) can be fulfilled for some vector $\mathbf{u}_{\Omega}$. Thus, $\lambda>2 d_{0}$ is the directional regime in the emission of photons, whereas in the case $\lambda<2 d_{0}$, we cannot ensure that photons are collimated in a single direction. In the following two subsections, we will study these regimes in 1D and 3D. In particular, we will be interested in determining $\Delta \theta$, the angular width of the photon beam in the directional regime, and $\Gamma_{\mathbf{n}}$, the collective emission rates. These quantities will be studied as a function of dimensionality, $d_{0}, \lambda$, and $N$.

\section{B. Atom chains}

We consider for concreteness that $\mathbf{k}_{\mathrm{L}}$ points in the $z$ direction, parallel to the chain axis. The vectors $\mathbf{n}$ and $\mathbf{m}$ are reduced now to scalars $n$ and $m$, which correspond to the projections on the chain axis. Due to the symmetry of the problem, the photon distribution emitted by a spin wave $n$ depends only on the angle $\theta$ through $\mathbf{u}_{\Omega}^{z}=\cos \theta$,

$$
I_{n}(\Omega)=\frac{1}{4 \pi} \frac{\bar{\Gamma}}{\Gamma_{n}} \frac{1}{N} \frac{\sin ^{2}\left[\left(k_{\mathrm{L}} d_{0}\left(\mathbf{u}_{\Omega}^{z}-1\right)-n \frac{2 \pi}{N}\right) N / 2\right]}{\sin ^{2}\left[\left(k_{\mathrm{L}} d_{0}\left(\mathbf{u}_{\Omega}^{z}-1\right)-n \frac{2 \pi}{N}\right) / 2\right]} .
$$

Taking the limit $N \gg 1$ and using (38),

$$
\begin{gathered}
I_{n}(\Omega)=\sum_{m} I_{n}^{[m]}(\Omega), \\
I_{n}^{[m]}(\Omega)=\frac{1}{4 \pi} \frac{\bar{\Gamma}}{\Gamma_{n}} f_{N}\left(\frac{k_{\mathrm{L}} d_{0}}{2}\left(\mathbf{u}_{\Omega}^{z}-1\right)-n \frac{\pi}{N}+m \pi\right) .
\end{gathered}
$$

The peaks in the photon distribution correspond to the emission of photons such that the $z$ component of momentum is conserved,

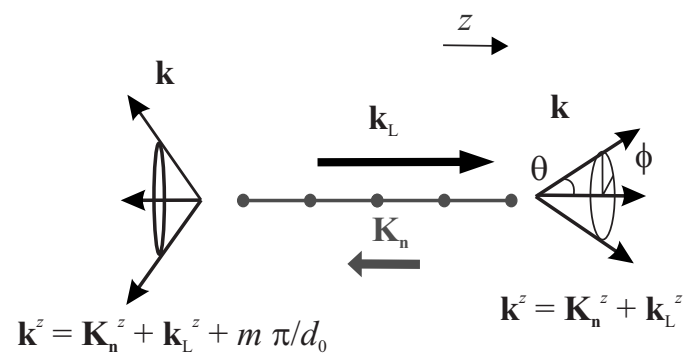

FIG. 2. Photon emission by a spin wave with linear momentum $\mathbf{K}_{n}$ in an atomic chain. The maxima in the photon distribution are at angles $\theta$ such that momentum along the chain is conserved up to reciprocal lattice wave vectors.

$$
\mathbf{u}_{\Omega}^{z}-1=\frac{\lambda}{d_{0}} \frac{n}{N}-\frac{\lambda}{d_{0}} m
$$

that is, there is a maximum whenever there is a value of $m$ which satisfies this relation. In the directional regime $\lambda$ $>2 d_{0}$, there is at most a single value of $m$ which satisfies (45). However, in this case, momentum conservation only determines the value of $\theta$ at the emission maximum, which implies that, in general, photons are emitted in cones spanned by different values of $\phi$. Only when the maximum happens at $\theta=0$ or $\theta=\pi$ are photons collimated in the forward- or backward-scattering directions, respectively (see Fig. 2).

Let us study first the case $n=0$ - that is, the emission properties of the completely symmetric state. The forwardscattering contribution $I_{0}^{[0]}$ has an angular width given by $\Delta \theta_{1 D}=1 / \sqrt{k_{\mathrm{L}} d_{0} N}$. The contribution to the emission pattern from each of the Bragg terms is

$$
\begin{gathered}
p_{0}^{[0]}=\int d \Omega I_{0}^{[0]}(\Omega)=\frac{\bar{\Gamma}}{\Gamma_{0}} \frac{\lambda}{4 d_{0}}, \\
p_{0}^{[m]}=2 p_{0}^{[0]}, \quad m \neq 0 .
\end{gathered}
$$

Condition (45) leads to the result that the number of emission cones with $m \neq 0$ is given by $\operatorname{int}\left(2 d_{0} / \lambda\right)$. A calculation made without resorting to the plane-wave approximation yields the same results, even for relatively small atom numbers $(N=20)$ (see Fig. 3). However, the shape of the photon angular distribution in the exact calculation shows a departure from the $\operatorname{sinc}^{2}$ shape predicted by Eq. (45) (see Fig. 4). By using the normalization condition for $I_{0}(\Omega)$, we determine the probability of emission in the forward-scattering direction,

$$
p_{0}^{[0]}=\frac{1}{1+2 \operatorname{int}\left(\frac{2 d_{0}}{\lambda}\right)} .
$$

Thus, in the directional regime $\lambda>2 d_{0}$, all the diffraction peaks but the forward-scattering one are suppressed. The collective emission rate is given by 


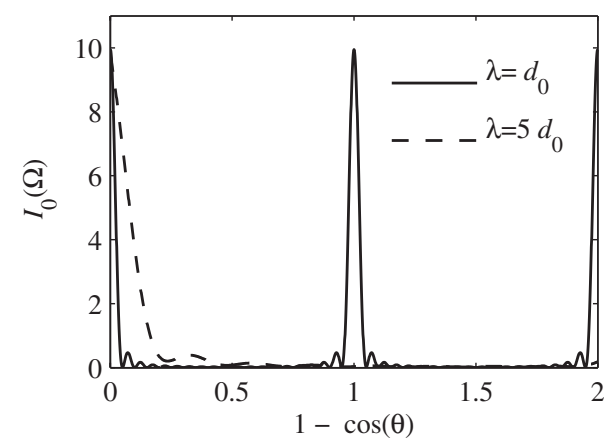

FIG. 3. Angular photon distribution of the photonic mode emitted by the completly symmetric spin wave in a chain of $N=20$ atoms. We have used the exact egenvalues of the density matrix and followed the method described in the previous section.

$$
\frac{\Gamma_{0}}{\bar{\Gamma}}=\frac{\lambda}{4 d_{0}}+\frac{\lambda}{2 d_{0}} \operatorname{int}\left(\frac{2 d_{0}}{\lambda}\right)
$$

where $\operatorname{int}(x)$ stands for the integer part of $x$.

Finally, we calculate the emission rate for all the 1D spinwave states. They can be written as an integration over the contributions coming from different diffraction peaks through the normalization condition; we have

$$
\frac{\Gamma_{n}}{\bar{\Gamma}}=\frac{1}{4 \pi} \sum_{m} \int_{-1}^{1} d x f_{N}\left(\frac{k_{\mathrm{L}} d_{0}}{2}(x-1)-n \frac{\pi}{N}+m \pi\right) .
$$

The collective rates are thus determined by the number of diffraction peaks which appear in the emission pattern of each collective state $n$. Considering the limit in which $k_{\mathrm{L}} d_{0} N \gg 1$ - that is, the $\delta$ limit for each of these peaks-we get the following distribution of emission rates:

$$
\frac{\Gamma_{n}}{\bar{\Gamma}}=\chi_{1 D}\left[\left(\theta\left(-\frac{n}{N}\right) \theta\left(\frac{2 d_{0}}{\lambda}+\frac{n}{N}\right)+\operatorname{int}\left(\frac{2 d_{0}}{\lambda}+\frac{n}{N}\right)\right],\right.
$$

with $\chi_{1 D}=\lambda /\left(2 d_{0}\right)$ and $\theta(x)$ is the Heaviside function. This expression describes quite well the emission rates calculated

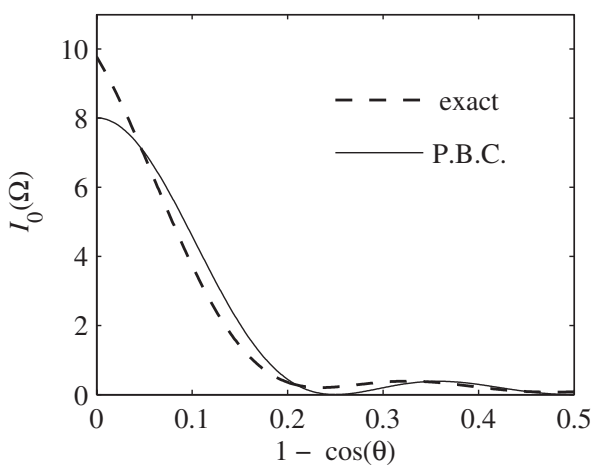

FIG. 4. Angular photon distribution of the photonic mode emitted by the completly symmetric spin wave from a chain of $N=20$ atoms, $\lambda=5 d_{0}$, calculated assuming periodic boundary conditions (solid line) and performing an exact diagonalization of the master equation (dashed line). The distribution in the exact case shows a departure from the $\operatorname{sinc}^{2}$ shape due to collective effects.

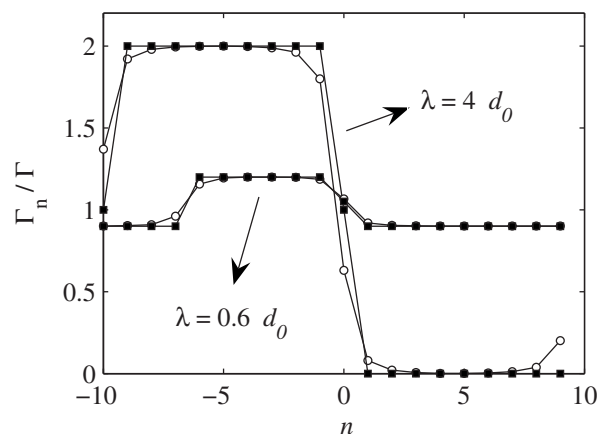

FIG. 5. Calculation of the collective rates of a chain with $N$ $=20$ atoms. Solid squares: result predicted by the plane-wave approximation (50). Open circles: eigenvalues of the matrix $J_{i j}$ defined by (14). In the last case, to each eigenvalue of $J_{i j}$ we assign a wave number $n$, which corresponds to the maximum Fourier component of the corresponding eigenvector.

without assuming periodic boundary conditions-that is, by diagonalizing the matrix $J_{i j}$ given by (14) (see Fig. 5). In the limit $d_{0} \gg \lambda$, we recover $\Gamma_{n}=\bar{\Gamma}$. On the contrary, if $\lambda$ is comparable to $d_{0}$, emission rates are renormalized. In the directional regime $\lambda>2 d_{0}$, some of the states have an enhanced rate $\Gamma_{n}=\chi_{1 D}$ (superradiant), whereas there are states for which $\Gamma_{n}=0$ (subradiant) [18]. Due to the relation between the photon distribution and the emission rates (49), subradiant states correspond to spin waves whose emission pattern is not peaked at a given value of $\theta$. On the contrary, superradiant states are spin waves whose emission pattern does contain a maximum as a function of $\theta$. Note that Eq. (50) does not describe the case of Dicke superradiance, which would predict a single superradiant state, with $\Gamma_{0}=N \bar{\Gamma}$. The reason is that we have assumed condition $N k_{\mathrm{L}} d_{0} \gg 1$; that is, we are always in the regime in which the light wavelength is much smaller than the size of the chain. Our results for 1D are consistent with Ref. [19], where the collective light emission from an atomic chain is addressed with the quantum jump formalism. Also, in Ref. [20], the fraction of subradiant states of a chain of interacting qubits is studied numerically, and the result is in agreement with Eq. (50).

To summarize the situation in $1 \mathrm{D}$, in the directional regime $\lambda>2 d_{0}$, superradiant spin waves emit photons into a single-emission cone with a rate that is enhanced by $\chi_{1 D}$. This effect can be used to generate photons that are collimated along the chain axis. Note that, in general, the atomphoton mapping induced with the $\Lambda$ scheme of Fig. 1 may compete with other radiative processes, such as the radiative decay from $|a\rangle$ back to $|s\rangle$ or the radiative decay from $|a\rangle$ to other atomic levels that are not included in the $\Lambda$ scheme. This problem can be solved by enhancing the atom-photon mapping rate, choosing $\chi_{1 D} \gg 1$. Since $\chi_{1 D}$ does not depend on the chain size, this implies choosing $\lambda \gg d_{0}$. Another way out of this problem is to use a cycling transition (see Appendix D).

\section{Three-dimensional atom lattices}

Contrary to the $1 \mathrm{D}$ case, in $3 \mathrm{D}$ it is not simple to obtain closed expressions to describe the emission in the plane- 
wave approximation. In order to get a simpler picture, we replace the sinc function in the definition (38) by a Gaussian which is normalized in the same way,

$$
f_{N}(x) \approx \sqrt{\pi} N_{x} e^{-x^{2} N^{2}} .
$$

This approximation is justified in the limit in which the diffraction peaks are narrow enough, such that they do not overlap-that is, $N_{\alpha} k_{\mathrm{L}} d_{0} \gg 1$. Assuming that the length of the lattice is the same in any spatial direction, $N_{x}=N_{y}=N_{z}$, then the angular photon distribution for a state $\mathbf{n}$ is given by

$$
\begin{gathered}
I_{\mathbf{n}}(\Omega)=\sum_{\mathbf{m}} I_{\mathbf{n}}^{[\mathbf{m}]}(\Omega), \\
I_{\mathbf{n}}^{[\mathbf{m}]}(\Omega)=\frac{1}{4 \pi} \frac{\bar{\Gamma}}{\Gamma_{\mathbf{n}}} \pi^{3 / 2} N e^{-\left[\left(k_{\mathrm{L}} d_{0} / 2\right) \mathbf{u}_{\Omega}-\mathbf{k}_{\mathrm{L}} d_{0} / 2-\pi \mathbf{n} / N-\pi \mathbf{m}\right]^{2} N_{x}^{2}} .
\end{gathered}
$$

A given term $I_{\mathbf{n}}^{[\mathbf{m}]}(\Omega)$ has a non-negligible contribution only if

$$
\frac{\left|\mathbf{k}_{\mathrm{L}} d_{0}+2 \pi \mathbf{n} / N+2 \pi \mathbf{m}\right|}{k_{\mathrm{L}} d_{0}}=1,
$$

since, otherwise, there are no photons which satisfy energymomentum conservation.

Let us study first the directional regime $\lambda>2 d_{0}$. In this case we find the following two possible situations.

(i) Superradiant states-spin waves for which there exists a value of $\mathbf{m}$, say, $\mathbf{m}_{c}$, such that (53) is satisfied. The condition $\lambda>2 d_{0}$ ensures that there is a single possible value of $\mathbf{m}_{c}$. Thus, the photon distribution can be simplified,

$$
I_{\mathbf{n}}(\Omega)=I_{\mathbf{n}}^{\left[\mathbf{m}_{c}\right]}(\Omega),
$$

and there is a single-emission peak in the direction of

$$
\mathbf{u}_{\Omega_{\max }}=\frac{\mathbf{k}_{\mathrm{L}} d_{0}+2 \pi \mathbf{n} / N+2 \pi \mathbf{m}_{c}}{k_{\mathrm{L}} d_{0}} .
$$

Photons are collimated in a beam with width $\Delta \theta_{3 D}$ $=1 /\left(k_{\mathrm{L}} d_{0} N^{1 / 3}\right)$. From (54) and the normalization condition, we can determine the emission rate,

$$
\begin{aligned}
\frac{\Gamma_{\mathbf{n}}}{\bar{\Gamma}} & =\frac{N}{4 \pi} \int d \Omega \pi^{3 / 2} e^{-\left[\left(k_{\mathrm{L}} d_{0} / 2\right) \mathbf{n}_{\Omega}-\mathbf{k}_{\mathrm{L}} d_{0} / 2-\pi \mathbf{n} / N-\pi \mathbf{m}_{c}\right]^{2} N_{x}^{2}} \\
& =\frac{1}{\sqrt{\pi}} N_{x}\left(\frac{\lambda}{2 d_{0}}\right)^{2}=\chi_{3 D} .
\end{aligned}
$$

Thus, superradiant states have a decay rate that is enhanced by the optical thickness, $\chi_{3 D}$.

(ii) Subradiant states-spin-waves for which there is no value of $\mathbf{m}$ which satisfies (53). In this case, there is not a single dominant contribution $\mathbf{m}$ such that directionality in the emission of photons is not guaranteed. Also, the normalization condition on $I_{\mathbf{n}}(\Omega)$ leads directly to the result $\Gamma_{\mathbf{n}}=0$ [18].

From this analysis, we conclude that superradiant spin waves are interesting in the context of quantum information, since they emit collimated photons with an enhanced emis- sion rate. However, the emission direction is determined by a value of $\mathbf{m}_{c}$, which has to be calculated for each particular spin wave. There are two relevant cases in which this situation becomes simpler, since energy-momentum conservation ensures that the only Bragg contribution is $\mathbf{m}_{\mathrm{c}}=\mathbf{0}$. The first one is the case $\lambda>4 d_{0}$, because

$$
\begin{gathered}
\left|k_{\mathrm{L}} \mathbf{u}_{\Omega}^{\alpha}-\mathbf{k}_{\mathrm{L}}^{\alpha}\right|<2 k_{\mathrm{L}}, \\
\left|\frac{n_{\alpha}}{N} \frac{2 \pi}{d_{0}}+m_{\alpha} \frac{2 \pi}{d_{0}}\right|>\frac{1}{2} \frac{2 \pi}{d_{0}} \quad(\text { if } \mathbf{m} \neq \mathbf{0}),
\end{gathered}
$$

and thus for values $\lambda>4 d_{0}$ the energy-momentum conservation condition (42) is only fulfilled by $\mathbf{m}_{\mathrm{c}}=\mathbf{0}$. Second, assume that the spin wave is created by the absorption of a photon within the $\Lambda$ configuration of Fig. 1, such that the spin-wave linear momentum can be written like $\mathbf{K}_{\mathbf{n}}=\mathbf{q}-\mathbf{k}_{\mathrm{L}}$, with $q=k_{\mathrm{L}}$. Then, the energy-momentum condition is now

$$
\mathbf{q}+\mathbf{k}=\mathbf{m} \frac{2 \pi}{d_{0}},
$$

which is satisfied only by $\mathbf{m}_{\mathrm{c}}=\mathbf{0}$ if $\lambda>2 d_{0}$. Indeed, the spin wave created in this way is always superradiant, since $\mathbf{K}_{\mathbf{n}}$ automatically satisfies Eq. (53). A similar result is obtained in Refs. [21,22] for the case of a cloud of atoms with a Gaussian density distribution, where the rate of spontaneous emission of a state created by the absorption of a photon is shown to increase with the optical thickness.

To summarize the situation in $3 \mathrm{D}$, in the directional regime $\lambda>2 d_{0}$, superradiant spin waves emit collimated photons with a rate enhanced by $\chi_{3 D}$. Contrary to the situation found in $1 \mathrm{D}$, in $3 \mathrm{D}$ lattices $\chi_{3 D} \propto N_{x}$, and thus it can be increased by increasing the size of the system. In this way, the atom-photon mapping could be much faster than other competing decay channels.

\section{EMISSION OF LIGHT BY HOT ATOMIC ENSEMBLES}

The formalism presented in the previous section can be extended to situations in which atoms are not at fixed positions in space. In this case, one expects the photonic state not to be a pure state, but a mixed one. This is relevant to the description of hot atomic vapors. We follow the discussion presented in Sec. II D, and consider the case of fast motion $\left(\tau_{\text {mot }} \ll \Gamma_{n}\right)$. The results presented here are in agreement with Ref. [23], where the photonic mode emitted by an atomic ensemble is studied. Our first task is to reformulate the problem of photon emission by finding the master equation that describes this situation.

\section{A. Master equation for atomic ensembles}

In the fast-motion limit, particle positions may be described as a set of independent random variables $\left\{\mathbf{r}_{j}\right\}$. We choose for simplicity a Gaussian distribution probability

$$
\rho(\mathbf{r})=\frac{1}{\pi^{3 / 2} L^{3}} e^{-(r / L)^{2}} .
$$

The particle positions fulfill the following identity, which will turn out to be the basis for the following calculations: 


$$
\left\langle e^{-i \mathbf{q} \cdot\left(\mathbf{r}_{j}-\mathbf{r}_{l}\right)}\right\rangle_{\mathrm{mot}}=\delta_{j l}+\left(1-\delta_{j l}\right) e^{-q^{2} L^{2} / 2},
$$

where $\langle\cdots\rangle_{\text {mot }}$ is an average over the atomic positions.

To calculate the coefficients of the master equation we start from Eq. (12) and perform the average,

$$
J_{i j}=\pi \sum_{\mathbf{k}} g_{k}^{2} \delta\left(\omega_{k}-\omega_{\mathrm{L}}\right)\left\langle e^{i\left(\mathbf{k}-\mathbf{k}_{\mathrm{L}}\right) \cdot\left(\mathbf{r}_{i}-\mathbf{r}_{j}\right)}\right\rangle_{\mathrm{mot}},
$$

were we have neglected the Cauchy principal value contribution, since it leads to an energy shift that does not play any role in the discussion that follows. After using (60), integrating over $\mathbf{k}$, and taking the limit $k_{\mathrm{L}} L \gg 1$, we get

$$
\begin{gathered}
J_{i i}=\bar{\Gamma} / 2, \\
J_{i j}=\frac{\bar{\Gamma}}{4\left(k_{\mathrm{L}} L\right)^{2}} \quad(i \neq j) .
\end{gathered}
$$

One can readly diagonalize the matrix $J_{i j}$ and obtain the eigenspaces of the master equation. The first is the completely symmetric state $(n=0)$,

$$
\mathcal{M}_{j 0}=\frac{1}{\sqrt{N}}, \quad J_{0}=\frac{\bar{\Gamma}}{2}\left(\chi_{\mathrm{en}}+1\right),
$$

where $\chi_{\mathrm{en}}=(N-1) /\left[2\left(k_{\mathrm{L}} L\right)^{2}\right]$ is the optical thickness of the atomic ensemble. Note that in the limit $N \gg 1$ and defining the atom density $n_{\mathrm{at}}=N / L^{3}$, the optical thickness can be recast in the more familiar form $\chi_{\mathrm{en}}=n_{\mathrm{at}} \chi^{2} L / 2$. The second eigenspace is spanned by the spin waves orthogonal to $\mathcal{M}_{j 0}$,

$$
\sum_{j} \mathcal{M}_{j n}=0, \quad J_{n}=\frac{\bar{\Gamma}}{2}\left(1-\frac{1}{2\left(k_{\mathrm{L}} L\right)^{2}}\right), \quad \text { if } n \neq 0 .
$$

Collective effects happen if $\chi_{\mathrm{en}} \gg 1$. In this case, there is a single superradiant spin-wave mode, corresponding to the completely symmetric state, and $N-1$ states which decay with the single-atom emission rate $J_{n}=\bar{\Gamma} / 2$.

\section{B. Single-photon state}

The spin-wave mode $n=0$ is the only one to show collective effects, and it is the collective state which can be created in experiments with atomic vapors. For these reasons, we focus on the radiation properties of these modes. First, we consider the emission of light by the initial atomic state with a single excitation,

$$
\left|\Psi_{0}\right\rangle_{\text {at }}=\frac{1}{\sqrt{N}} \sum_{j} b_{j}^{+}|0\rangle_{\text {at }} .
$$

The atom-photon mapping can be extended to this situation by considering first that the atomic state is mapped onto a given photonic state and then by performing the average over the atomic positions. This gives as a result a photon density matrix

$$
\begin{gathered}
\left|\Psi_{0}\right\rangle_{\mathrm{at}}|0\rangle_{\mathrm{ph}}\langle 0|\rightarrow| 0\rangle_{\mathrm{at}} \rho, \\
\rho=\sum_{\mathbf{k}, \mathbf{k}^{\prime}} \rho_{\mathbf{k k}^{\prime}} a_{\mathbf{k}}^{+}|0\rangle_{\mathrm{ph}}\langle 0| a_{\mathbf{k}^{\prime}} .
\end{gathered}
$$

Note that the mapping is now from a pure to a mixed state. Unfortunately, with the statistical properties of the atomic positions considered here, it is not possible to ensure that this is still a Gaussian map. We can, however, use Eqs. (24) and (60) to get

$$
\begin{gathered}
\rho_{\mathbf{k} \mathbf{k}^{\prime}}=\left\langle\phi_{0, \mathbf{k}} \phi_{0, \mathbf{k}^{\prime}}^{*}\right\rangle_{\mathrm{mot}}=(1-\epsilon) \bar{\phi}_{0, \mathbf{k}} \bar{\phi}_{0, \mathbf{k}^{\prime}}^{*}+\epsilon \bar{\rho}, \\
\epsilon=\frac{1}{1+\chi_{\mathrm{en}}} .
\end{gathered}
$$

We work, for clarity, in the interaction picture with respect to $H_{1 \mathrm{~m}}$. The coherent component of the photon density matrix is the pure state $\left|\bar{\phi}_{0}\right\rangle_{\mathrm{ph}}$,

$$
\bar{\phi}_{0, \mathbf{k}}=\sqrt{1+\frac{1}{\chi_{\mathrm{en}}}} \sqrt{N-1} g_{k} \frac{e^{-\left|\mathbf{k}-\mathbf{k}_{\mathrm{L}}\right|^{2} L^{2} / 4}}{-i\left(\omega_{k}-\omega_{\mathrm{L}}\right)+J_{0}},
$$

whereas $\bar{\rho}$ is a normalized mixed state which describes the incoherent (isotropic) component,

$$
\bar{\rho}_{\mathbf{k k}^{\prime}}=\frac{\left(1+\chi_{\mathrm{en}}\right) g_{k} g_{k^{\prime}} e^{-\left|\mathbf{k}-\mathbf{k}^{\prime}\right|^{2} L^{2} / 4}}{\left[-i\left(\omega_{k}-\omega_{\mathrm{L}}\right)+J_{0}\right]\left[i\left(\omega_{k^{\prime}}-\omega_{\mathrm{L}}\right)+J_{0}\right]} .
$$

Under the condition $\chi_{\mathrm{en}} \gg 1$, the main contribution to the photon density matrix is the pure one $\left|\phi_{0}\right\rangle_{\mathrm{ph}}$. The parameters which describe the directionality in the photon emission can be readly evaluated. The emission pattern has both coherent and incoherent contributions

$$
\begin{gathered}
I_{0}(\Omega)=I_{0}^{\mathrm{coh}}(\Omega)+I_{0}^{\mathrm{inc}}(\Omega), \\
I_{0}^{\mathrm{coh}}(\Omega)=(1-\epsilon) \frac{N-1}{4 \pi \chi_{\mathrm{en}}} e^{-\left(k_{\mathrm{L}} \mathbf{u}_{\Omega}-\mathbf{k}_{\mathrm{L}}\right)^{2} L^{2} / 2}, \\
I_{0}^{\mathrm{inc}}(\Omega)=\epsilon \frac{1}{4 \pi} .
\end{gathered}
$$

Thus, photons are collimated in the forward-scattering direction, with angular width $\Delta \theta_{\mathrm{en}}=1 /\left(k_{\mathrm{L}} L\right)$. The deviation of the photon emission from directionality is determined by the probability of emission out of the forward-scattering cone, $\mathcal{E}=\int d \Omega I_{0}^{\text {inc }}(\Omega)=\epsilon$.

Finally, we calculate the purity of the photon state, which is defined as

$$
\mathcal{P}=\operatorname{Tr}\left(\rho^{2}\right)=(1-\epsilon)^{2}+\epsilon^{2} \operatorname{Tr}\left(\bar{\rho}^{2}\right)+2 \epsilon(1-\epsilon)\left\langle\bar{\phi}_{0}|\bar{\rho}| \bar{\phi}_{0}\right\rangle .
$$

This quantity is very relevant when using photons in quantum information processing, since it describes the efficiency in the process of interference of photonic modes in a beam splitter, being $\mathcal{P}=1$, the case corresponding to a pure state. To calculate $\mathcal{P}$, we notice first that 


$$
\operatorname{Tr}\left(\bar{\rho}^{2}\right)=\sum_{\mathbf{k}, \mathbf{k}^{\prime}}\left|\rho_{\mathbf{k}, \mathbf{k}^{\prime}}\right|^{2}=\frac{1}{2\left(k_{\mathrm{L}} L\right)^{2}} .
$$

Also, because of the Cauchy-Schwartz inequality, this implies that

$$
\left|\left\langle\phi_{0}|\bar{\rho}| \phi_{0}\right\rangle\right|^{2} \leqslant \operatorname{Tr}\left(\bar{\rho}^{2}\right),
$$

such that

$$
\mathcal{P}=\operatorname{Tr}\left(\rho^{2}\right)=(1-\epsilon)^{2}+O\left(1 / k_{\mathrm{L}} L\right) \approx 1-2 / \chi_{\mathrm{en}} .
$$

Thus, in the limit $k_{\mathrm{L}} L \gg 1$, the purity of the photon state is solely determined by $\chi_{\mathrm{en}}$.

\section{Multiphoton state}

We study now the properties of the multiphoton case. The mapping is from a state with $M$ atomic excitations in the completely symmetric state to a multiphoton mixed state,

$$
\frac{1}{\sqrt{M !}}\left(\frac{1}{\sqrt{N}} \sum_{j} b_{j}^{\dagger}\right)^{M}|0\rangle_{\mathrm{at}}|0\rangle_{\mathrm{ph}} \rightarrow|0\rangle_{\mathrm{at}} \rho .
$$

We assume the low-excitation limit $(M \ll N)$. In Appendix B, we show that the mixed photonic state can be written like

$$
\begin{gathered}
\rho=(1-\epsilon)^{M}|\Phi\rangle_{\mathrm{ph}}\langle\Phi|+\tilde{\rho}, \\
|\Phi\rangle_{\mathrm{ph}}=\frac{1}{\sqrt{M !}}\left(\sum_{\mathbf{k}} \bar{\phi}_{0, \mathbf{k}} a_{\mathbf{k}}^{+}\right)^{M}|0\rangle_{\mathrm{ph}} .
\end{gathered}
$$

That is, it is a sum of a pure state consisting of $M$ photons in the photonic mode defined by (68) and the (not normalized) mixed state $\tilde{\rho}$. In order to determine the purity of $\rho$ we should study the mixed contribution, which is here far more complicated than in the single-photon case. Instead, we notice that

$$
\mathcal{P}=\operatorname{Tr}\left(\rho^{2}\right) \geqslant(1-\epsilon)^{2 M},
$$

which allows us to obtain a lower bound for the purity.

\section{IMPLEMENTATIONS}

\section{A. Collective light emission in ultracold atoms in optical lattices}

Ultracold atoms in optical lattices are an ideal system for the observation of the effects described in this work. For example, atoms in a Mott phase [24] would be ideally suited to study collective light emission from a square lattice. In this setup, atoms are placed at distances that are comparable to optical wavelengths, since potential wells in a standing wave are indeed separated by $d_{0}=\lambda_{\mathrm{sw}} / 2$, with $\lambda_{\mathrm{sw}}$ the wavelength of the counterpropagating lasers that create the lattice. The conditions for the directional regime are met by using an optical transition such that $\lambda>\lambda_{\mathrm{sw}}$. Under this condition, ultracold atoms in optical lattices are ideal to form an atomlight quantum interface $[11,25,26]$, where the quantum state of light can be indeed manipulated, as shown recently in [27]. The properties of the emitted light may also be used to (i)
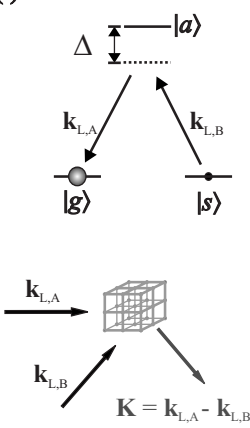

(ii)
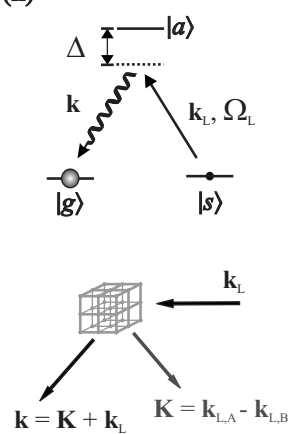

FIG. 6. (i) Creation of a spin wave with linear momentum $\mathbf{k}_{\mathrm{L}, \mathrm{A}}+\mathbf{k}_{\mathrm{L}, \mathrm{B}}$ in an optical lattice. (ii) Mapping from the spin wave to a photonic mode.

measure the properties of quantum many-body phases in optical lattices [28]. Our theory can also be applied to the collective matter-wave emission in optical lattices proposed in Ref. [29].

We propose now an experiment in which the renormalization of the collective rates may be observed by performing the following steps (see Fig. 6).

(i) First, we use a protocol to initialize the atomic quantum state of the lattice. Assume that all the atoms are initially in the ground state $|g\rangle$. The $\Lambda$ scheme of Fig. 1 may be used for the initialization by shining the lattice during a short time $T$ with two lasers with wave vectors $\mathbf{k}_{\mathrm{L}, \mathrm{A}}, \mathbf{k}_{\mathrm{L}, \mathrm{B}}$, and Rabi frequencies $\Omega_{\mathrm{L}, \mathrm{A}}, \Omega_{\mathrm{L}, \mathrm{B}}$, and detuning $\Delta$, such that $\Omega_{\mathrm{L}, \mathrm{A}}, \Omega_{\mathrm{L}, \mathrm{B}} \ll \Delta$. Under these conditions, a coherent state of spin waves with momentum $\mathbf{K}=\mathbf{k}_{\mathrm{L}, \mathrm{A}}-\mathbf{k}_{\mathrm{L}, \mathrm{B}}$ is created,

$$
\begin{gathered}
|\Psi\rangle_{\mathrm{at}} \propto e^{-i\left(\Omega_{\mathrm{AB}} T / 2\right) b_{K}^{\dagger}}|0\rangle_{\mathrm{at}}, \\
b_{\mathbf{K}}^{\dagger}=\frac{1}{\sqrt{N}} \sum_{j} e^{i \mathbf{K} \cdot \mathbf{r}_{j}} b_{j}^{\dagger},
\end{gathered}
$$

where $\Omega_{\mathrm{AB}}=\Omega_{\mathrm{L}, \mathrm{A}} \Omega_{\mathrm{L}, \mathrm{B}} / \Delta$ and the condition $\Omega_{\mathrm{AB}} T \ll 1$ has to hold in order to ensure that the system is in the lowexcitation regime.

(ii) After the creation of the collective atomic state, this can be realeased by a second laser with momentum $\mathbf{k}_{\mathrm{L}}$ in the $\Lambda$ scheme considered along this work. The analysis presented in Sec. III can be applied to study the emission of photons by setting $\mathbf{K}_{\mathbf{n}}=\mathbf{k}_{\mathrm{L}, \mathrm{A}}-\mathbf{k}_{\mathrm{L}, \mathrm{B}}$. For example, if $\mathbf{k}_{\mathrm{L}}$ $=\mathbf{k}_{\mathrm{L}, \mathrm{B}}$, then the spin wave emits a photon in the direction $\mathbf{k}_{\mathrm{L}, \mathrm{A}}$, with a superradiant rate $\chi_{3 D} \bar{\Gamma}$. On the contrary, if $\mathbf{K}_{\mathbf{n}}$ does not satisfy the condition (53), then the emission rate will be suppressed, up to finite-size effects.

\section{B. Deterministic generation of entangled states of photons}

An application of our ideas to generate photons in a deterministic way requires a system where experimentalists are able both to reach the directional regime of collective light emission and to initialize the atomic system in a given spinwave state. This idea may find useful applications in quantum cryptography [30], quantum computation [31], and quantum litography [32]. 


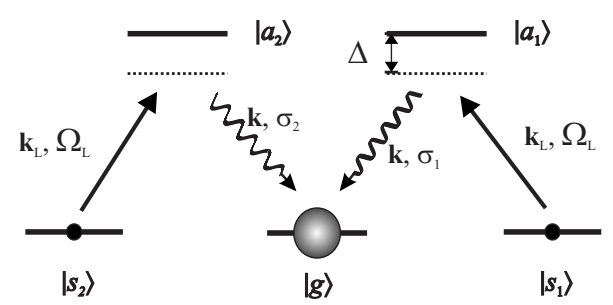

FIG. 7. Double- $\Lambda$ scheme for the generation of photons entangled in polarization.

Before going into the description of particular experimental setups, we discuss how to extend our formalism to generate photons that are entangled in polarization. For this, we consider the double- $\Lambda$ scheme of Fig. 7, in which there are two excited levels $\left|s_{1}\right\rangle,\left|s_{2}\right\rangle$ and a ground-state level $|g\rangle$. The atom-photon mapping is described in the same way, by defining the corresponding Holstein-Primakoff operators $b_{1, j}$, $b_{2, j}$. Assume that the $\left|s_{1}\right\rangle,\left|s_{2}\right\rangle$ states decay by emitting photons with different polarizations and creation operators $a_{1, \mathbf{k}}^{\dagger}$, $a_{2, \mathbf{k}}^{\dagger}$. If the conditions for momentum conservation explained along this work are fulfilled, then entangled spin waves will be mapped onto entangled photons in polarization. For example, consider the initial spin-wave state

$$
|\Psi\rangle_{\mathrm{at}}=\frac{1}{\sqrt{2}}\left(b_{1, \mathbf{K}_{\mathrm{A}}}^{\dagger} b_{2, \mathbf{K}_{\mathrm{B}}}^{\dagger}+b_{1, \mathbf{K}_{\mathrm{B}}}^{\dagger} b_{2, \mathbf{K}_{\mathrm{A}}}^{\dagger}\right)|0\rangle_{\mathrm{at}},
$$

where we have used the notation $b_{\sigma, \mathbf{K}}^{\dagger}=(1 / \sqrt{N}) \sum_{j} e^{i \mathbf{K r}_{j}} b_{\sigma, j}^{\dagger}$. After the atom-photon mapping, the following photonic state is created:

$$
|\Phi\rangle_{\mathrm{ph}}=\frac{1}{\sqrt{2}}\left(a_{1, \mathbf{K}_{\mathrm{A}}+\mathbf{k}_{\mathrm{L}}}^{\dagger} a_{2, \mathbf{K}_{\mathrm{A}}+\mathbf{k}_{\mathrm{L}}}^{\dagger}+a_{1, \mathbf{K}_{\mathrm{B}}+\mathbf{k}_{\mathrm{L}}}^{\dagger} a_{2, \mathbf{K}_{\mathrm{A}}+\mathbf{k}_{\mathrm{L}}}^{\dagger}\right)|0\rangle_{\mathrm{ph}} .
$$

In this way, the ability to generate entangled spin waves is equivalent, by virtue of the atom-photon mapping, to the ability to generate entangled photonic states. We propose three experimental setups where this is possible and discuss the conditions that are required for the implementation of this idea.

\section{Trapped ions}

This system is especially appealing from the point of view of the creation of entangled states, since quantum gates allow us to create deterministically any collective state. This idea has been demonstrated in the creation of the $\mathbf{K}=\mathbf{0}$ (W) state in [4]. The first issue that we have to deal with is whether conditions for forward scattering of photons hold here. Usually ions are arranged in chains, with the peculiarity that the distance between ions is not constant. However, if condition $d_{0}^{\text {av }}<\lambda / 2$, with $d_{0}^{\text {av }}$ the average distance, we still get light emission in the forward-scattering cone only. We show this fact by performing a calculation of the emission pattern of a single atomic excitation in the $\mathbf{K}=\mathbf{0}$ state using the theory presented in the previous sections (see Fig. 8). Note that directionality is achieved even for a relatively small number of ions $(N=10)$. The main difficulty for the implementation

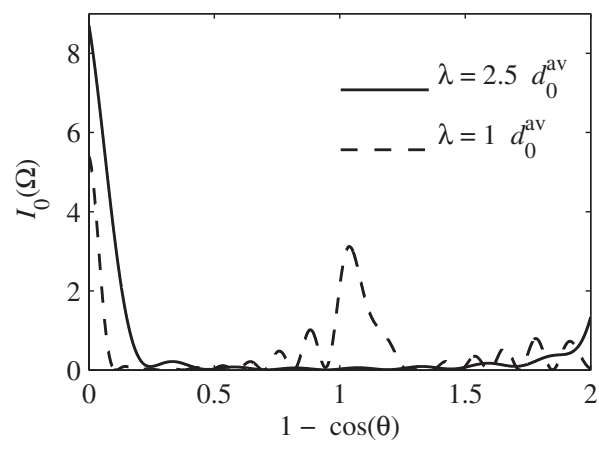

FIG. 8. Angular photon distribution of the photonic mode emitted by the completely symmetric spin wave in an ion chain with $N=10$ ions.

of this idea with ions lies in the fact that ion-ion distances are usually in the range of a few $\mu \mathrm{m}$, and thus condition $d_{0}^{\text {av }}$ $<\lambda / 2$ is not fulfilled when considering optical wavelengths [33]. A way out of this problem is to use optical transitions which lie in the range of $\lambda \gtrsim 5 \mu \mathrm{m}$, such as, for example, $\lambda\left({ }^{2} D_{3 / 2}-{ }^{2} P_{1 / 2}\right)=10.8 \mu \mathrm{m}$ in $\mathrm{Hg}^{+}$or $\lambda\left({ }^{2} D_{3 / 2}-{ }^{2} D_{5 / 2}\right)$ $=12.5 \mu \mathrm{m}$ in $\mathrm{Ba}^{+}$. A particularly interesting initial atomic state is the spin wave (79) with $\mathbf{k}_{\mathrm{A}}=\mathbf{0}$ and $\mathbf{k}_{\mathrm{B}}=-2 \mathbf{k}_{\mathrm{L}}$ (all vectors in the direction of the atomic chain), since it leads to the emission of an entangled photon pair in the forward- and backward-emission directions.

\section{Ultracold atoms in optical lattices}

We have shown above that optical lattices are well suited to reach the regime of directionallity in the emission of photons. The main issue here, contrary to the case of trapped ions, is to find a way to create efficiently the initial spinwave states in a deterministic way. Although one could think of peforming quantum gates between ultracold neutral atoms to generate collective atomic states $[34,35]$, this procedure faces the difficulties of quantum computation in this system, such as, for example, how to achieve single-atom addressability.

More efficiently, one could avoid the use of quantum gates by using the dipole-blockade mechanism with Rydberg atoms, which allows us to generate $\mathrm{W}$ states, as well as states which emit Fock states with a number $M$ of photons [2]. Interactions between excited atomic states, such as those that take place in Rydberg atoms, can be also used to generate photons entangled in polarization. This can be achieved in a single experimental step, without the need for quantum gates, if the proper configuration of atomic interactions is chosen. As an example, consider the level configuration shown in Fig. 9 and interactions between excited states such that atoms in levels $\left|s_{1}\right\rangle$ and $\left|s_{2}\right\rangle$ interact strongly only if they are in the same excited state-that is, $U_{11}=U_{22}=U$, but $U_{12}=0$. We apply two lasers with wave vectors $\mathbf{k}_{\mathrm{A}, \mathrm{B}}$ and Rabi frequencies $\Omega_{\mathrm{A}, \mathrm{B}}$, detuned with respect to the $|g\rangle$ $-\left|s_{a, b}\right\rangle$ transition, such that $\Delta_{\mathrm{A}}=-\Delta_{\mathrm{B}}=\Delta$. If condition $\Delta_{\mathrm{A}, \mathrm{B}}$ $\gg \Omega_{\mathrm{A}, \mathrm{B}}$ is fulfilled, then the lasers induce a two-photon transition with Rabi frequency $\Omega_{\text {eff }}=\Omega_{\mathrm{A}} \Omega_{\mathrm{B}} / \Delta$. Furthermore, if $\Omega_{\text {eff }} \ll U$, states with two atoms in the same excited state are not populated. Under these conditions there are two possible 


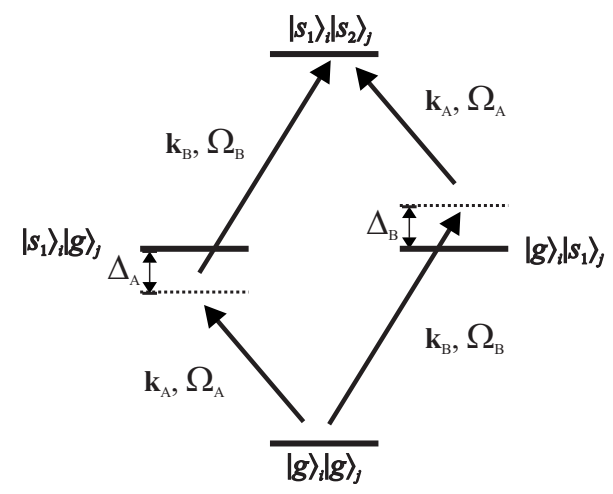

FIG. 9. Scheme for the generation of entangled spin waves by using the Rydberg blockade.

excitation channels, depicted in Fig. 9, which give rise to the linear combination (79).

\section{Atomic ensembles at room temperature}

The very same techniques which can be applied to Rydberg atoms in an optical lattice can also be used in the case of hot ensembles. On the one hand, this setup has the advantage that atoms do not need to be cooled and placed in an optical lattice. On the other hand, it can be described by a statistical distribution of particles and thus suffers from the fact that high efficiency in the release of photons is achieved under more severe conditions of particle density and atom number, as discussed above. However, densities which are high enough to fulfill the requirement $\mathcal{E} \ll 1$ have been recently reported in [36].

\section{Photon up-conversion}

Finally, the ideas presented in this work find an intereseting application in the efficient up-conversion of photons. For example, transitions between the states $|g\rangle$ and $|s\rangle$ are typically in the range of $1 \mathrm{GHz}$. Application of the $\Lambda$ scheme of Fig. 1 provides a way for up-conversion of the microwave photon to an optical photon. Also, by using several lasers, one could induce $N$-photon transitions from $|g\rangle$ to $|a\rangle$ and emit a single high-frequency photon. By using the ideas presented in this work, this could be done in such a way that the up-converted photons are collimated and, thus, they can be efficiently collected.

\section{ACKNOWLEDGMENTS}

We acknowledge interesting discussions with M. Lewenstein. Work supported by the EU (SCALA) and the DFG through the excellence cluster Munich Advance Photonics (MAP).

\section{APPENDIX A: VALIDITY OF NEGLECTING PROPAGATION EFFECTS}

When performing the integration in $k$ in Eq. (27), we have to deal with the following factor inside the integration:

$$
\frac{e^{i\left(\mathbf{k}-\mathbf{k}_{\mathrm{L}}\right) \cdot\left(\mathbf{r}_{j}-\mathbf{r}_{j^{\prime}}\right)}}{\left[-i\left(\omega_{k}-\omega_{\mathrm{L}}\right)-\bar{J}_{n}^{*}\right]\left[i\left(\omega_{k}-\omega_{\mathrm{L}}\right)-\bar{J}_{n^{\prime}}\right]},
$$

which can be evaluated in the following way:

$$
\begin{aligned}
\frac{1}{\left[-i\left(\omega_{k}-\omega_{\mathrm{L}}\right)-\bar{J}_{n}^{*}\right]\left[i\left(\omega_{k}-\omega_{\mathrm{L}}\right)-\bar{J}_{n^{\prime}}\right]} \\
=-\left(\frac{1}{-i\left(\omega_{k}-\omega_{\mathrm{L}}\right)-\bar{J}_{n}^{*}}+\frac{1}{i\left(\omega_{k}-\omega_{\mathrm{L}}\right)-\bar{J}_{n^{\prime}}}\right) \\
\quad \times\left(\bar{J}_{n}^{*}+\bar{J}_{n^{\prime}}\right)^{-1} \\
\approx-2 \pi \delta\left(\omega_{k}-\omega_{L}\right) \frac{1}{\bar{J}_{n}^{*}+\bar{J}_{n^{\prime}}},
\end{aligned}
$$

where we have aproximated

$$
\operatorname{Re}\left(\frac{1}{-i\left(\omega_{k}-\omega_{\mathrm{L}}-\Delta_{n}\right)-\Gamma_{n} / 2}\right) \approx \pi \delta\left(\omega_{k}-\omega_{\mathrm{L}}\right) .
$$

This approximation is justified by the following argument. The left-hand side of (A2) is a function of $k$ with width $\Gamma_{n} / c$. The latter has to be compared with the dependence on momentum of the exponential in (A1), which is, roughly, $1 / L$. Thus, the approximation holds in the limit $\Gamma_{n} / c \ll 1 / L$ - that is, whenever the emission time is shorter than the propagation of the photon through the sample, something that is well justified in the trapping setups considered in this work. The energy shift can also be safely neglected, since condition $\Delta_{n} \ll \omega_{L}$ is well justified by the atomic transitions used in cold-atom setups.

\section{APPENDIX B: MULTIPHOTON STATE EMITTED BY AN ATOMIC ENSEMBLE}

Our starting point is the atom-photon mapping defined in Eq. (75). By applying our method we get

$$
\begin{aligned}
\rho= & \frac{1}{M !} \frac{1}{N^{M}}\left\langle\left(\sum_{j, \mathbf{k}} \frac{e^{i\left(\mathbf{k}-\mathbf{k}_{\mathrm{L}}\right) \cdot \mathbf{r}_{j}}}{i\left(\omega_{k}-\omega_{\mathrm{L}}\right)-\Gamma_{0} / 2} a_{\mathbf{k}}^{\dagger}\right)^{M} \mid 0\right\rangle_{\mathrm{ph}}\langle 0| \\
& \left.\times\left(\sum_{j^{\prime}, \mathbf{k}^{\prime}} \frac{e^{-i\left(\mathbf{k}^{\prime}-\mathbf{k}_{\mathrm{L}}\right) \cdot \mathbf{r}_{j^{\prime}}}}{-i\left(\omega_{k^{\prime}}-\omega_{\mathrm{L}}\right)-\Gamma_{0} / 2} a_{\mathbf{k}^{\prime}}\right)^{M}\right\rangle_{\mathrm{mot}} .
\end{aligned}
$$

After expanding the contents of the two parentheses to the power of $M$ we can reexpress this equation as a sum of $2 M$ products of exponentials of the argument $i\left(\mathbf{k}-\mathbf{k}_{\mathrm{L}}\right) \mathbf{r}_{j}$. There are $\left(\begin{array}{c}N \\ 2 M\end{array}\right)$ terms such that all the random variables are different. These terms can be summed up to a contribution which yields a pure state, such that $\rho$ is the sum of a pure and a mixed state,

$$
\rho=\tilde{\rho}_{\text {pure }}+\tilde{\rho},
$$

where $\tilde{\rho}_{\text {pure }}$ and $\tilde{\rho}$ are not normalized. The pure contribution has the form 


$$
\begin{aligned}
\tilde{\rho}_{\text {pure }}= & \frac{1}{M !} \frac{1}{N^{M}}\left(\begin{array}{c}
N \\
2 M
\end{array}\right)\left(\sum_{\mathbf{k}} \frac{g_{k} e^{-\left|\mathbf{k}-\mathbf{k}_{\mathrm{L}}\right|^{2} L^{2} / 4}}{i\left(\omega_{k}-\omega_{\mathrm{L}}\right)-\Gamma_{0} / 2} a_{\mathbf{k}}^{\dagger}\right)^{M}|0\rangle_{\mathrm{ph}}\langle 0| \\
& \times\left(\sum_{\mathbf{k}^{\prime}} \frac{g_{k^{\prime}} e^{-\left|\mathbf{k}^{\prime}-\mathbf{k}_{\mathrm{L}}\right|^{2} L^{2} / 4}}{-i\left(\omega_{k^{\prime}}-\omega_{\mathrm{L}}\right)-\Gamma_{0} / 2} a_{\mathbf{k}^{\prime}}\right)^{M},
\end{aligned}
$$

which is a pure state with $M$ photons. To normalize this state, we use the limit $\left(\begin{array}{c}N \\ 2 M\end{array}\right) / N^{M}=N^{M}(N \gg M)$ and obtain the final result (76).

\section{APPENDIX C: 3D LATTICE, LIMIT $\lambda \ll d_{0}$}

In the case of atoms in a 3D square lattice, in the limit $\lambda \ll d_{0}$, there are in principle many diffraction peaks which contribute to the emission pattern. In the following we show that, at least for the completely symmetric state, if the size of the system is large enough, the forward-scattering contribution is the most important one.

Recall the definition of the probability that the spin wave $\mathbf{0}$ emits a photon in the $\mathbf{m}$ Bragg scattering peak, $p_{\mathbf{0}}^{[\mathbf{m}]}$, Eq. (41). The forward-scattering contribution $\mathbf{m}=\mathbf{0}$ is given by

$$
p_{0}^{[0]}=\frac{\bar{\Gamma}}{\Gamma_{0}} \chi_{3 D} .
$$

We consider for concreteness $\mathbf{k}_{\mathrm{L}}=k_{\mathrm{L}} \hat{z}$. To determine the probability of scattering out of the forward direction, $\mathcal{E}$, we calculate the sum over the contributions with $\mathbf{m} \neq 0$,

$$
\begin{aligned}
\mathcal{E} & =\sum_{\mathbf{m} \neq 0} p_{0}^{[\mathbf{m}]} \\
& =\frac{\bar{\Gamma}}{\Gamma_{0}} \sum_{\mathbf{m} \neq 0} \frac{N}{4 \pi} \int d \Omega \pi^{3 / 2} e^{-\left[\mathbf{u}_{\Omega}-\hat{z}-\left(\lambda / d_{0}\right) \mathbf{m}\right]^{2}\left(k_{\mathbf{L}} d_{0} N_{x} / 2\right)^{2}} .
\end{aligned}
$$

Considering the limit $k_{\mathrm{L}} d_{0} N_{x} / 2 \gg 1$, the angular integral yields

$$
\mathcal{E}=\sum_{\mathbf{m} \neq 0} \frac{\bar{\Gamma}}{\Gamma_{0}} \pi^{3 / 2} \frac{N}{\left(k_{\mathrm{L}} d_{0} N_{x}\right)^{2}} e^{-\left[1-\mid\left(\lambda / d_{0}\right) \mathbf{m}+\hat{z}\right]^{2}\left(k_{\mathrm{L}} d_{0} N_{x} / 2\right)^{2}}
$$

In the limit $d_{0} \gg \lambda$, we can replace the sum by an integration over $\mathbf{m}$ and get the result

$$
\mathcal{E}=\frac{\bar{\Gamma}}{\Gamma_{0}} .
$$

Together with the normalization condition $\Sigma_{\mathbf{m}} p_{\mathbf{m}}=1$, this leads to

$$
\mathcal{E}=\frac{1}{1+\chi_{3 D}}, \quad \frac{\Gamma_{0}}{\bar{\Gamma}}=1+\chi_{3 D} .
$$

Thus, as long as the optical thickness is large, the completely symmetric state is superradiant and photons are collimated in

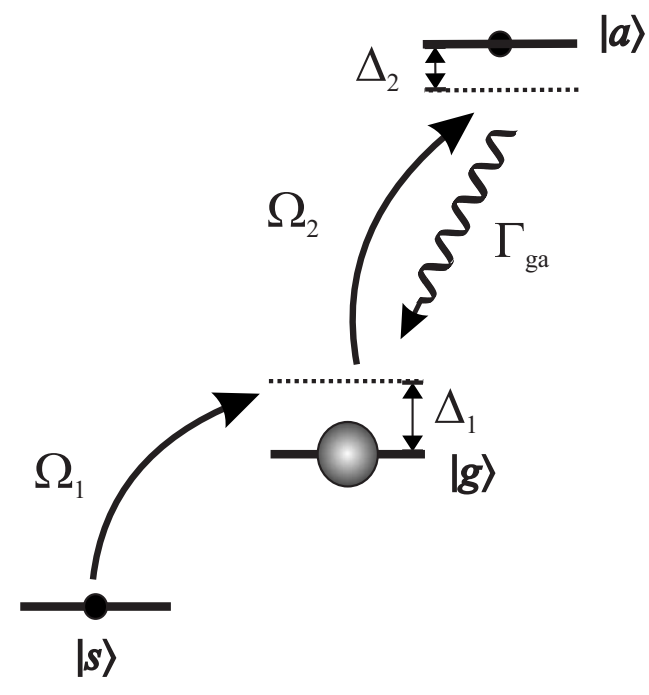

FIG. 10. Level scheme for the implementation of the atomphoton mapping, avoiding incoherent processes.

the forward-scattering direction. Note that the regime $\lambda$ $\ll d_{0}$ is very different than the directional regime, in the sense that there is always a nonzero probability of emission out of the forward-scattering cone. In the fully directional regime $\left(\lambda>2 d_{0}\right)$, on the contrary, $\mathcal{E}=0$, up to finite-size effects.

\section{APPENDIX D: CYCLING TRANSITION}

Up to now we have neglected the decay form the auxiliary level $|a\rangle$ back to the excited state $|s\rangle$. This process can be suppressed by considering, for example, a level configuration like the one presented in Fig. 10.

$|s\rangle$ and $|g\rangle$ could be two hyperfine states of the electronic ground-state manifold and $|a\rangle$ be chosen such that $|a\rangle-|g\rangle$ is a cycling transition. For example, these levels could be found in ${ }^{87} \mathrm{Rb}:|s\rangle \rightarrow\left|S_{1 / 2}, F=1, m_{F}=1\right\rangle,|g\rangle \rightarrow\left|S_{1 / 2}, F=2, m_{F}=2\right\rangle$, and $|a\rangle \rightarrow\left|P_{1 / 2}, F=3, m_{F}=3\right\rangle$. Spontaneous emission from $|a\rangle$ to $|s\rangle$ is forbidden by selection rules on $m_{F}$, but atoms decay from $|a\rangle$ to $|g\rangle$ with a rate $\Gamma_{\text {ga }}$. Fields with Rabi frequencies $\Omega_{1}, \Omega_{2}$, and detunings $\Delta_{1}, \Delta_{2}$, are coupled to the transitions $|s\rangle-|g\rangle,|g\rangle-|s\rangle$, respectively. Under some conditions, lasers induce a two-photon transition that is equivalent to a $\Lambda$ configuration such as the one considered in this work with $\Omega_{\mathrm{L}}$ $=\Omega_{\text {eff }}=\Omega_{1} \Omega_{2} / \Delta_{1}$.

We consider two situations.

(i) $\Delta_{2} \gg \Gamma_{\mathrm{ga}}$. The atom-photon mapping is possible under the conditions $\Omega_{\mathrm{eff}} \ll \Delta_{2}$ (adiabatic elimination of the level $|a\rangle$ ), $\sqrt{N} \Omega_{1} \ll \Delta_{1}$ (to avoid real transitions from $|s\rangle$ to $|g\rangle$ ), and $\Gamma_{\mathrm{ga}}\left(\Omega_{\mathrm{eff}} / \Delta_{2}\right)^{2} \gg N \Gamma_{\mathrm{ga}}\left(\Omega_{2} / \Delta\right)^{2}$, or equivalently, $\Omega_{1} / \Delta_{2} \gg \sqrt{N}$, to avoid the dephasing induced by the transfer of atoms from $|g\rangle$ to $|a\rangle$.

(ii) $\Delta_{2}=0$ (resonant case). Conditions: $\Omega_{\mathrm{eff}} \ll \Gamma_{\text {ga }}$ (adiabatic elimination of $|a\rangle) . \Omega_{1} / \Delta_{2} \gg \sqrt{N}$, and $\Gamma_{\mathrm{ga}}\left(\Omega_{\mathrm{eff}} / \Gamma_{\mathrm{ga}}\right)^{2}$ $\gg N \Gamma_{\text {ga }}\left(\Omega_{2} / \Delta_{1}\right)$, or equivalently, $\Omega_{1}^{2} \gg N \Gamma_{\text {ga }}^{2}$ [same reasons as (i)]. 
[1] D. Leibfried et al., Nature (London) 438, 639 (2004); F. Schmidt-Kaler et al., ibid. 422, 408 (2003).

[2] M. D. Lukin, M. Fleischhauer, R. Cote, L.-M. Duan, D. Jaksch, J. I. Cirac, and P. Zoller, Phys. Rev. Lett. 87, 037901 (2001).

[3] D. Tong, S. M. Farooqi, J. Stanojevic, S. Krishnan, Y. P. Zhang, R. Cote, E. E. Eyler, and P. L. Gould, Phys. Rev. Lett. 93, 063001 (2004); K. Singer, M. Reetz-Lamour, T. Amthor, L. G. Marcassa, and M. Weidemüller, ibid. 93, 163001 (2004).

[4] H. Häffner et al., Nature (London) 438, 643 (2004).

[5] D. Porras and J. I. Cirac, e-print arXiv:0704.0641.

[6] R. H. Dicke, Phys. Rev. 93, 99 (1954).

[7] M. O. Scully, E. S. Fry, C. H. Raymond Ooi, and K. Wódkiewicz, Phys. Rev. Lett. 96, 010501 (2006).

[8] T. Holstein and H. Primakoff, Phys. Rev. 58, 1098 (1940).

[9] E. Ressayre and A. Tallet, Phys. Rev. A 11, 981 (1975).

[10] M. Fleischhauer and M. D. Lukin, Phys. Rev. Lett. 84, 5094 (2000).

[11] K. Hammerer, A. S. Sorensen, and E. S. Polzik, e-print arXiv:0807.3358.

[12] W. Żakowicz and K. Rzazewski, J. Phys. A 7, 869 (1974); W. Żakowicz, Phys. Rev. A 17, 343 (1978).

[13] G. Giedke and J. I. Cirac, Phys. Rev. A 66, 032316 (2002).

[14] The rotating-wave approximation is applied to derive Eq. (3). However, counterrotating terms must be included to get correctly the dipole-dipole contribution to the master equation coefficients. The inclusion of counterrotating terms does not change the mapping between Fock states of spin waves to photons.

[15] R. H. Lehmberg, Phys. Rev. A 2, 883 (1970).

[16] See, e. g., J. D. Jackson, Classical Electrodynamics (Wiley, New York, 1962).

[17] J. H. Eberly, J. Phys. B 39, S599 (2006).

[18] Since this result is obtained under the assumption of periodic boundary conditions, subradiant states in finite lattices will have a small nonzero decay rate due to finite-size corrections.

[19] J. P. Clemens, L. Horvath, B. C. Sanders, and H. J. Carmichael, Phys. Rev. A 68, 023809 (2003).
[20] C. J. Mewton and Z. Ficek, J. Phys. B 40, S181 (2007).

[21] I. E. Mazets and G. Kurizki, J. Phys. B 40, F105 (2007).

[22] A. A. Svidzinsky, J.-T. Chang, and M. O. Scully, Phys. Rev. Lett. 100, 160504 (2008).

[23] L.-M. Duan, J. I. Cirac, and P. Zoller, Phys. Rev. A 66, 023818 (2002).

[24] M. Greiner, O. Mandel, T. Esslinger, T. Hänsch, and I. Bloch, Nature (London) 415, 39 (2002).

[25] C. W. Chou, H. de Riedmatten, D. Felinto, S. V. Polyakov, S. J. van Enk, and H. J. Kimble, Nature (London) 438, 828 (2005); T. Chaneliare, D. N. Matsukevich, S. D. Jenkins, S.-Y. Lan, T. A. B. Kennedy, and A. Kuzmich, ibid. 438, 833 (2005); M. D. Eisaman, A. Andrè, F. Massou, M. Fleischhauer, A. S. Zibrov, and M. D. Lukin, ibid. 438, 837 (2005).

[26] L.-M. Duan, M. D. Lukin, J. I. Cirac, and P. Zoller, Nature (London) 414, 413 (2001).

[27] C. A. Muschik, I. de Vega, D. Porras, and J. I. Cirac, Phys. Rev. Lett. 100, 063601 (2008).

[28] I. de Vega, J. I. Cirac, and D. Porras, Phys. Rev. A 77, 051804(R) (2008).

[29] I. de Vega, D. Porras, and J. I. Cirac, e-print arXiv:0807.1901.

[30] Nicolas Gisin, Grégoire Ribordy, Wolfgang Tittel, and Hugo Zbinden, Rev. Mod. Phys. 74, 145 (2002).

[31] E. Knill, R. Laflamme, and G. J. Milburn, Nature (London) 409, 46 (2001).

[32] A. N. Boto, P. Kok, D. S. Abrams, S. L. Braunstein, C. P. Williams, and J. P. Dowling, Phys. Rev. Lett. 85, 2733 (2000).

[33] The renormalization of the emission rates in systems of two ions has been studied and shown experimentally. See R. G. Brewer, Phys. Rev. Lett. 77, 5153 (1996); R. G. DeVoe and R. G. Brewer, ibid. 76, 2049 (1996).

[34] D. Jaksch, J. I. Cirac, P. Zoller, S. L. Rolston, R. Cote, and M. D. Lukin, Phys. Rev. Lett. 85, 2208 (2000).

[35] O. Mandel, M. Greiner, A. Widera, T. Rom, T. W. Hänsch, and I. Bloch, Nature (London) 425, 937 (2003).

[36] R. Heidemann, U. Raitzsch, V. Bendkowsky, B. Butscher, R. Löw, L. Santos, and T. Pfau, Phys. Rev. Lett. 99, 163601 (2007). 